\title{
MULTIWAVELENGTH OBSERVATIONS OF RADIO-QUIET QUASARS WITH WEAK EMISSION LINES
}

\author{
Richard M. Plotkin ${ }^{1,2}$, Scott F. Anderson ${ }^{2}$, W. N. Brandt ${ }^{3,4}$, Aleksandar M. Diamond-Stanic ${ }^{5}$, Xiaohui Fan ${ }^{5}$, \\ Chelsea L. Macleod ${ }^{2}$, Donald P. Schneider ${ }^{3}$, and Ohad Shemmer ${ }^{6}$ \\ ${ }^{1}$ Astronomical Institute “Anton Pannekoek," University of Amsterdam, Science Park 904, 1098 XH, Amsterdam, The Netherlands; r.m.plotkin@uva.nl \\ 2 Department of Astronomy, University of Washington, Box 351580, Seattle, WA 98195, USA \\ ${ }^{3}$ Department of Astronomy and Astrophysics, Pennsylvania State University, 525 Davey Laboratory, University Park, PA 16802, USA \\ ${ }^{4}$ Institute for Gravitation and the Cosmos, The Pennsylvania State University, University Park, PA 16802, USA \\ 5 Steward Observatory, University of Arizona, Tucson, AZ 85721, USA \\ ${ }^{6}$ Department of Physics, University of North Texas, Denton, TX 76203, USA \\ Received 2010 June 10; accepted 2010 July 26; published 2010 August 30
}

\begin{abstract}
We present radio and X-ray observations, as well as optical light curves, for a subset of $26 \mathrm{BL}$ Lac candidates from the Sloan Digital Sky Survey (SDSS) lacking strong radio emission and with $z<2.2$. Half of these 26 objects are shown to be stars, galaxies, or absorbed quasars. We conclude that the other 13 objects are active galactic nuclei (AGNs) with abnormally weak emission features; 10 of those 13 are definitively radio quiet, and, for those with available optical light curves, their level of optical flux variability is consistent with radio-quiet quasars. We cannot exclude the possibility that some of these 13 AGNs lie on the extremely radio-faint tail of the BL Lac distribution, but our study generally supports the notion that all BL Lac objects are radio-loud. These radio-quiet AGNs appear to have intrinsically weak or absent broad emission line regions (BELRs), and, based on their X-ray properties, we argue that some are low-redshift analogs to weak line quasars (WLQs). SDSS BL Lac searches are so far the only systematic surveys of the SDSS database capable of recovering such exotic low-redshift WLQs. There are 71 more $z<2.2$ radio-quiet BL Lac candidates already identified in the SDSS, but not considered here, and many of those might be best unified with WLQs as well. Future studies combining low- and high-redshift WLQ samples will yield new insight on our understanding of the structure and formation of AGN BELRs.
\end{abstract}

Key words: BL Lacertae objects: general - quasars: emission lines

Online-only material: color figure

\section{INTRODUCTION}

Strong optical and ultraviolet (UV) emission lines are among the most prominent and often, defining characteristics of active galactic nuclei (AGNs). The rare instances of AGN lacking strong emission features are typically classified as BL Lacertae objects. In addition to their featureless optical spectra, BL Lac objects are strong radio, X-ray, and gamma-ray emitters, they are highly polarized, and they display strong and rapid flux variability across the entire electromagnetic spectrum (e.g., see Kollgaard 1994; Perlman et al. 2001). In the standard unification paradigm, BL Lac objects are explained as low-luminosity radio galaxies with a relativistic jet pointed toward the observer (e.g., see Blandford \& Rees 1978; Urry \& Padovani 1995). In this scenario, BL Lac objects' emission lines are diluted by the Doppler boosted relativistic jet, and the BL Lac phenomenon should go hand in hand with strong radio emission. Observations have indeed established that radio-quiet BL Lac objects must be extraordinarily rare if they exist at all (e.g., see Jannuzi et al. 1993; Londish et al. 2004). For example, Stocke et al. (1990) found no examples of radio-quiet BL Lac objects in the X-ray-selected BL Lac sample from the Einstein Observatory Extended Medium-Sensitivity Survey (EMSS; Stocke et al. 1991).

It is possible, however, that venerable BL Lac samples such as the EMSS were merely too small ( $\sim 40$ objects) to reveal especially rare subsets of radio-shy BL Lac objects. With large-scale multiwavelength surveys and inclusive selection approaches, the number of known BL Lac objects has grown over the past $10-15$ years, from a couple hundred cataloged in Padovani \& Giommi (1995b) to $\sim 10^{3}$ in Massaro et al. (2009). Quasar samples have also become extraordinarily large; for example, the Sloan Digital Sky Survey (SDSS; York et al. 2000) Data Release Seven (DR7) Quasar Catalog contains $\sim 10^{5}$ quasars with reliable spectroscopic redshifts (Schneider et al. 2010). These quasar samples are now large enough to reveal interesting examples of rare quasar populations, such as radio-quiet objects with weak or absent spectral features, in numbers comparable to early BL Lac samples. For example, Collinge et al. (2005, hereafter C05) and Anderson et al. (2007, hereafter A07) discovered about two dozen BL Lac candidates lacking strong radio emission. Plotkin et al. (2010, hereafter P10) present a sample of 723 optically selected BL Lac objects uniformly selected from the SDSS that includes 86 objects with radio fluxes or limits firmly placing them in the radio-quiet regime.

This population of weak-featured radio-quiet objects recovered by SDSS BL Lac searches is intriguing, and it is puzzling how nature can create weak-featured AGNs that are not strong radio emitters. If these objects' emission lines are not simply diluted by beamed emission, then some other mechanism must be invoked to explain their apparently anemic broad emission line regions (BELRs).

Around one-third of the weak-featured radio-quiet AGNs recovered by SDSS BL Lac searches have $z>2.2$. These high-redshift objects pass the phenomenological criteria to alternatively be classified as weak line quasars (WLQs) - a rare class of high-redshift AGNs discovered by the SDSS with weak or absent UV emission features (see Fan et al. 1999; Anderson et al. 2001; Shemmer et al. 2006, 2009; DiamondStanic et al. 2009). There is significant overlap in the optical properties of WLQs and BL Lac objects, but WLQs tend to be weaker radio and X-ray emitters and they show less flux 
Table 1

Radio-quiet BL Lac Candidates

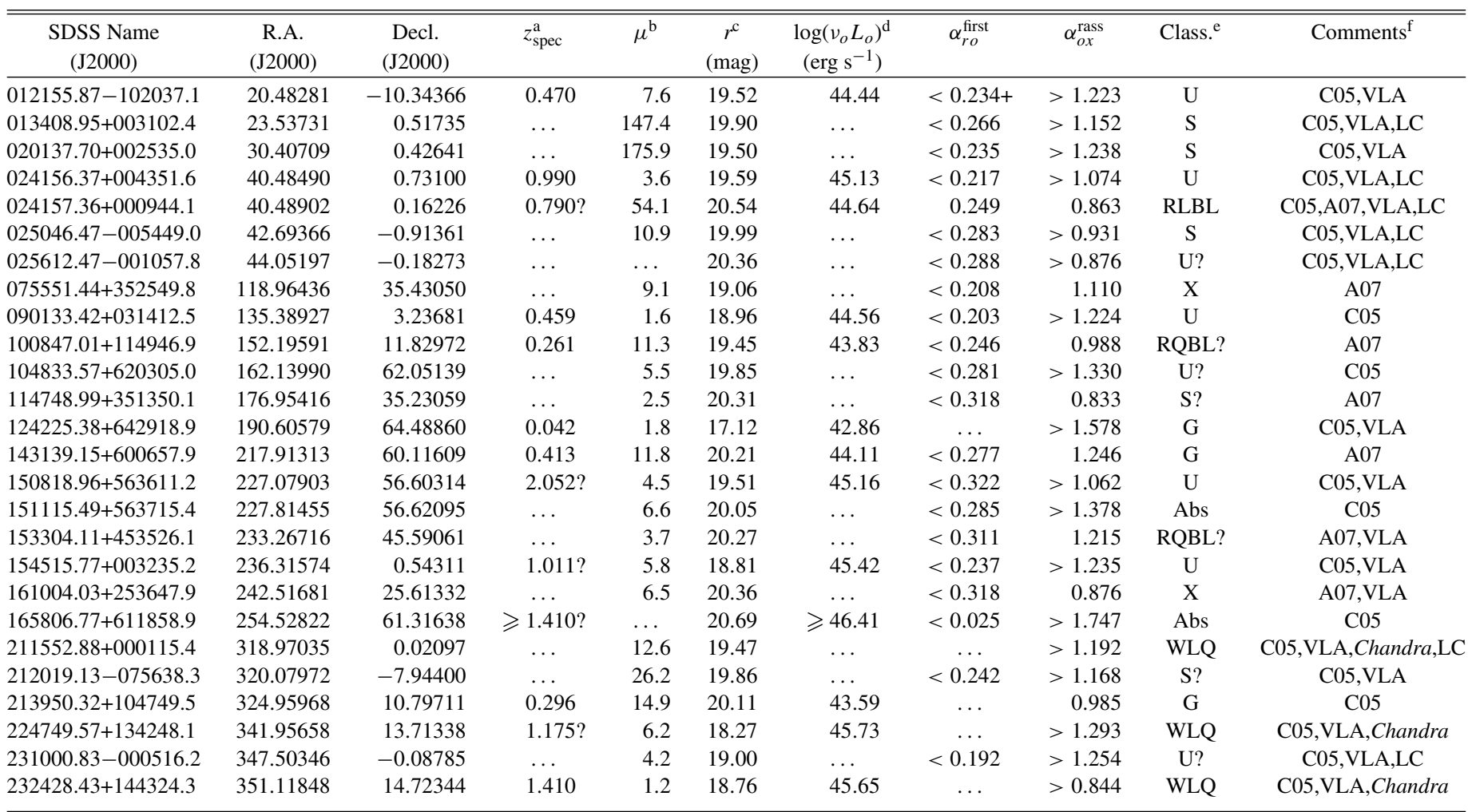

Notes.

a Tentative redshifts marked with "?". All objects have redshift upper limits $z<2.2$, derived by the fact that the Lyman forest is not seen in their SDSS spectra. If an object lacks a redshift, we assume $z=0.3$ when calculating $\alpha_{r o}^{\text {first }}$ and $\alpha_{o x}^{\text {rass }}$.

$\mathrm{b}$ In mas $\mathrm{yr}^{-1}$, from Munn et al. (2004). We consider proper motion measures to be significantly large if $\mu>30 \mathrm{mas}^{-1} \mathrm{because}^{-1}$ only $1 \%$ of spectroscopically confirmed SDSS quasars have such large proper motion measures (see Section 4.2).

${ }^{c}$ Point-spread function magnitude in the SDSS $r$ filter.

d At rest frame $5000 \AA$.

e Our classification (see Sections 4 and 5). Radio-quiet weak-featured AGNs are classified as radio-quiet BL Lac objects (RQBL?), low-redshift WLQs (WLQ), or unknown (U). Contaminants (i.e., objects unlikely to be AGN with intrinsically weak lines) are classified as absorbed AGNs (Abs), galaxies (G), radio-loud BL Lac objects (RLBL), stars (S), or they are unlikely the proper optical counterpart to an RASS X-ray source (X). Question marks denote low-confidence classifications.

${ }^{f}$ C05: listed as a radio-quiet BL Lac candidate in Collinge et al. (2005); A07: X-ray-selected BL Lac candidate lacking radio emission in Anderson et al. (2007); VLA: we took follow-on $8.4 \mathrm{GHz}$ radio observations with the VLA (see Section 3.1); Chandra: we took follow-on X-ray observations with Chandra (see Section 3.2); LC: we have a light curve from SDSS Stripe 82 (see Section 3.3).

variability and polarization. WLQs appear to compose an exotic population of featureless AGNs distinct from BL Lac objects (e.g., see Shemmer et al. 2006, 2009; Diamond-Stanic et al. 2009). That is, they seem to have intrinsically weak BELRs, and their emission lines are unlikely to be simply diluted by a beamed relativistic jet.

WLQ redshifts can often be determined only from the onset of the Ly $\alpha$ forest in their SDSS spectra. For this reason, studies that explicitly search for WLQs in the SDSS database only target high-redshift sources, and $\sim 80$ high-redshift WLQs have been discovered by the SDSS to date. SDSS BL Lac searches are sensitive not only to high-redshift WLQs but also to lowerredshift analogs, if such objects exist. ${ }^{7}$ Perhaps some of the lower-redshift weak-featured radio-quiet AGNs from SDSS BL Lac searches are actually lower-redshift analogs to high-redshift WLQs.

In this paper, we discuss the multiwavelength properties of the population of 26 lower-redshift $(z<2.2)$ radio-quiet BL Lac candidates recovered by the C05 and A07 surveys.

\footnotetext{
7 BL Lac surveys do not recover all known WLQs because BL Lac searches
} typically impose more stringent criteria on spectral feature strengths.
We briefly discuss objects from P10 as well, but we defer a detailed description of their properties for another paper. Throughout, we refer to these objects as "radio-quiet BL Lac candidates" to reflect the method by which they were discovered, but we will show that not all objects should be unified with normal radio-loud BL Lac objects. In Section 2, we present the sample of 26 low-redshift radio-quiet BL Lac candidates. We then present new radio and X-ray observations of a selected subset in Sections 3.1 and 3.2, respectively. In Section 3.3, we discuss their optical variability, and we compare them to radio-loud BL Lac objects as well as to normal radio-quiet quasars. Contaminants are identified in Section 4. In Section 5, we discuss potential physical interpretations for their weakfeatured spectra; we compare them to other lineless AGNs in the literature. Our main conclusions are summarized in Section 6. We adopt the following cosmology: $H_{0}=71 \mathrm{~km} \mathrm{~s}^{-1} \mathrm{Mpc}^{-1}$, $\Omega_{m}=0.27$, and $\Lambda_{0}=0.73$.

\section{THE PARENT RADIO-QUIET BL Lac SAMPLE}

In Table 1, we present all 26 low-redshift $(z<2.2)$ radioquiet BL Lac candidates recovered by $\mathrm{C} 05$ and A07. Throughout 
the text we identify objects that should be removed from this list (also see Section 4), but we include all 26 objects here for completeness. Sources with $z>2.2$ are not included in Table 1 because WLQs have been discussed elsewhere (e.g., Shemmer et al. 2009; Diamond-Stanic et al. 2009).

We refer the reader to $\mathrm{C} 05$ and $\mathrm{A} 07$ for detailed descriptions of their BL Lac selection. Briefly, both surveys require objects with optical SDSS spectra to not show any emission features with rest-frame equivalent widths (REWs) stronger than $5 \AA$, and all spectra must also show a $\mathrm{Ca}$ II $\mathrm{H} / \mathrm{K}$ depression smaller than $40 \%$ (see Landt et al. 2002). A07 require the additional constraint that all BL Lac candidates must match within $60^{\prime \prime}$ to an $\mathrm{X}$-ray source in the ROSAT All Sky Survey (RASS; Voges et al. 1999, 2000), with no constraints on their radio emission. C05 do not explicitly require radio or X-ray emission for inclusion; post-selection, their sample is correlated to RASS in the X-ray and to the Faint Images of the Radio Sky at Twenty-cm survey (FIRST; Becker et al. 1995) and to the NRAO VLA Sky Survey (NVSS; Condon et al. 1998) in the radio. Both radio surveys were performed with the Very Large $\operatorname{Array}^{8}$ (VLA) at $1.4 \mathrm{GHz}$. The 20 radio-quiet BL Lac candidates from $\mathrm{C} 05$ are not detected in the radio by FIRST/NVSS (or are not in the FIRST footprint), or they have radio detections but are radio-quiet. Seven objects from A07 lack radio detections in FIRST/NVSS, and one A07 object was also identified as a radio-quiet BL Lac candidate by $\mathrm{C} 05$.

The parameters listed in Table 1 are taken from the SDSS DR7 database (Abazajian et al. 2009). We adopt the spectroscopic redshift from the literature, unless we can identify a better redshift from visual examination of their DR7 spectral reductions. Of the 26 objects, 13 have spectroscopic redshifts identified from weak spectral features. Five of the thirteen redshifts are tentative (i.e., they are less certain because their weak emission line identifications are less secure or they show only one emission line that we assume to be $\mathrm{Mg}$ II), which we treat as exact. One of these tentative redshifts is actually a lower limit derived from possible intervening $\mathrm{Mg}$ II absorption. All objects in Table 1 have redshift upper limits $z<2.2$, or else the Lyman forest would be observed in their SDSS spectra. We also list morphology and proper motion information in Table 1 to help assess if any objects lacking redshifts might be stars. Proper motions are taken from the SDSS+USNO-B proper motion catalog (Munn et al. 2004), as listed in the SDSS DR7 database.

Table 1 includes broadband radio to optical $\left(\alpha_{\text {ro }}\right)$ and optical to X-ray $\left(\alpha_{\text {ox }}\right)$ spectral indices for each entry. ${ }^{9}$ The $\alpha_{\text {ro }}$ values in Table 1 are based on FIRST detections (or limits) and SDSS, and the $\alpha_{\text {ox }}$ values are derived from RASS and SDSS. We assume that objects with unknown redshifts have $z=0.3$ and redshift lower limits are exact, but we note that $\alpha_{\text {ro }}$ and $\alpha_{\text {ox }}$ are relatively insensitive to the exact redshift used. Blank entries for $\alpha_{\text {ro }}$ indicate that the source is outside the FIRST footprint. C05 and A07 use slightly different parameters for estimating broadband spectral indices, so we recalculate them here. If contamination from the host galaxy to the SDSS optical spectrum is observed, then we decompose the SDSS spectrum

\footnotetext{
8 The National Radio Astronomy Observatory is a facility of the National Science Foundation operated under cooperative agreement by Associated Universities, Inc.

9 The broadband spectral index, for $v_{2}>v_{1}$, is defined as $\alpha_{\nu_{1} \nu_{2}}=-\log \left(L_{v_{2}} / L_{v_{1}}\right) / \log \left(\nu_{2} / \nu_{1}\right)$. Here, $\alpha_{\text {ro }}=-\log \left(L_{o} / L_{r}\right) / 5.08$ and $\alpha_{\text {ox }}=-\log \left(L_{x} / L_{o}\right) / 2.60$, where $L_{r}, L_{o}$, and $L_{x}$ are the specific luminosities (per unit frequency) at rest frames $5 \mathrm{GHz}, 5000 \AA$, and $1 \mathrm{keV}$, respectively (Tananbaum et al. 1979; Stocke et al. 1985).
}

into an elliptical galaxy ${ }^{10}$ (using the template of Mannucci et al. 2001) and a power law to measure each object's decomposed AGN optical flux (see P10) and its optical spectral index $\alpha_{o}$ (all local spectral indices are defined as $f_{v} \sim v^{-\alpha_{v}}$ ). We use these $\alpha_{o}$ measures, and we assume radio and X-ray spectral indices of $\alpha_{r}=-0.27$ and $\alpha_{x}=1.25$, respectively, to calculate $\alpha_{\text {ro }}$ and $\alpha_{\text {ox }}$ at rest frames $5 \mathrm{GHz}, 5000 \AA$, and $1 \mathrm{keV}$. Limits on radio luminosities are estimated for sources not detected in the radio by FIRST/NVSS by assuming radio flux densities $f_{1.4 \mathrm{GHz}}<0.25+5 \sigma_{\text {rms }} \mathrm{mJy}$, where 0.25 is the CLEAN bias and $\sigma_{\text {rms }}$ is the uncertainty of the FIRST survey at the source's location on the sky (see Becker et al. 1995). We derive flux density limits from FIRST rather than NVSS because of its higher angular resolution. The optical magnitude in the SDSS filter closest to rest frame $5000 \AA$ is used for calculating optical luminosities (and we correct for extinction with the Schlegel et al. 1998 dust maps). X-ray count rate limits are estimated for $\mathrm{X}$-ray-undetected sources as six counts divided by the exposure time of the nearest RASS X-ray source. ${ }^{11}$ We then calculate X-ray luminosities (or limits) at $1 \mathrm{keV}$ using the Portable, Interactive Multi-Mission Simulator (PIMMS; Mukai 1993) correcting for Galactic extinction with the Stark et al. (1992) H I maps.

Throughout, we consider objects with $\alpha_{\text {ro }}<0.2$ to be radioquiet for two reasons: (1) this is consistent with the traditional definition for the radio-quiet AGN, that is, radio to optical flux ratios $R<10$ (Kellermann et al. 1989; Stocke et al. 1992). (2) There are 285 optically selected SDSS BL Lac objects from P10 with both X-ray and radio detections in RASS and FIRST/NVSS; these follow a Gaussian distribution in $\alpha_{\text {ro }}$ with $\left\langle\alpha_{\text {ro }}\right\rangle=0.42 \pm 0.08$. Thus, any object with $\alpha_{\text {ro }}<0.2$ is significantly different from a BL Lac object in its radio properties at the $>2.75 \sigma$ level ( $>99.7 \%$ one-sided significance). This implicitly accounts for the non-simultaneity of our radio and optical observations, and the fact that we assume the same radio spectral index for every object. We note this is slightly conservative because SDSS BL Lac objects detected by RASS are among the $\mathrm{X}$-ray brightest and are radio-weaker on average (see Plotkin et al. 2008).

\section{MULTIWAVELENGTH PROPERTIES}

\subsection{Radio Observations with the VLA}

We obtained radio observations with the VLA for 17/26 radio-quiet BL Lac candidates from $\mathrm{C} 05$ and $\mathrm{A} 07$. These objects were targeted because they are among the optically brightest candidates $(g<21.3)$, and their FIRST/NVSS radio flux limits are not sensitive enough to place them in the radio-quiet regime (or they do not fall within the FIRST footprint). Due to scheduling constraints, 5/26 C05/A07 $g<21.3$ radio-quiet BL Lac candidates falling between $07^{\mathrm{h}}<$ R.A. $<12^{\mathrm{h}}$ were not observed. The remaining 4/26 C05/A07 objects were either too optically faint $(g>21.3)$ to efficiently obtain improved $\alpha_{\text {ro }}$ constraints with the VLA, or they were already known to have $\alpha_{\text {ro }}<0.2$ from their FIRST radio flux limits.

VLA observations were taken in the D-array configuration on 2007 March 30 for 5 targets, and in the DnC-array configuration

\footnotetext{
${ }^{10}$ BL Lac objects are probably exclusively hosted by elliptical galaxies (e.g., see Urry et al. 2000). Host galaxy contamination can typically be seen if the host galaxy accounts for $\gtrsim 20 \%$ of the observed flux.

11 For the undetected RASS sources, we did not find adequate archived pointed X-ray observations (i.e., from SWIFT, Chandra, XMM-Newton, etc.).
} 
Table 2

VLA Observations at $8.4 \mathrm{GHz}$ of Radio-quiet BL Lac Candidates

\begin{tabular}{|c|c|c|c|c|c|c|c|}
\hline $\begin{array}{l}\text { SDSS Name } \\
(\mathrm{J} 2000)\end{array}$ & Redshift & Config $^{\mathrm{a}}$ & $\begin{array}{c}\sigma_{\mathrm{rms}} \\
\left(\mathrm{mJy} \mathrm{beam}^{-1}\right)\end{array}$ & $\begin{array}{c}f_{\text {vla }} \\
(\mathrm{mJy})\end{array}$ & $\alpha_{\mathrm{ro}}^{\mathrm{vla}}$ & $\alpha_{\mathrm{ro}}^{\mathrm{first}}$ & $\alpha_{\mathrm{Ox}}^{\mathrm{rass}}$ \\
\hline $012155.87-102037.1$ & 0.470 & $\mathrm{DnC}$ & 0.033 & $\ldots$ & $<0.038$ & $<0.234$ & $>1.223$ \\
\hline $013408.95+003102.4$ & $\ldots$ & $\mathrm{DnC}$ & 0.047 & $\ldots$ & $<0.123$ & $<0.266$ & $>1.152$ \\
\hline $020137.70+002535.0$ & $\ldots$ & $\mathrm{DnC}$ & 0.032 & $\ldots$ & $<0.058$ & $<0.235$ & $>1.238$ \\
\hline $024156.37+004351.6$ & 0.990 & $\mathrm{DnC}$ & 0.036 & 0.43 & 0.109 & $<0.217$ & $>1.074$ \\
\hline $024157.36+000944.1$ & $0.790 ?$ & $\mathrm{DnC}$ & 0.041 & 2.44 & 0.318 & 0.249 & 0.863 \\
\hline $025046.47-005449.0$ & $\ldots$ & $\mathrm{DnC}$ & 0.033 & $\ldots$ & $<0.095$ & $<0.283$ & $>0.931$ \\
\hline $025612.47-001057.8$ & $\ldots$ & $\mathrm{DnC}$ & 0.032 & $\ldots$ & $<0.111$ & $<0.288$ & $>0.876$ \\
\hline $124225.38+642918.9$ & 0.042 & $\mathrm{DnC}$ & 0.035 & $\ldots$ & $<-0.063$ & $\ldots$ & $>1.578$ \\
\hline $150818.96+563611.2$ & $2.052 ?$ & $\mathrm{DnC}$ & 0.032 & $\ldots$ & $<0.128$ & $<0.322$ & $>1.062$ \\
\hline $153304.11+453526.1$ & $\ldots$ & $\mathrm{DnC}$ & 0.036 & $\ldots$ & $<0.133$ & $<0.311$ & 1.215 \\
\hline $154515.77+003235.2$ & $1.011 ?$ & $\mathrm{DnC}$ & 0.041 & $\ldots$ & $<-0.009$ & $<0.237$ & $>1.235$ \\
\hline $161004.03+253647.9$ & $\ldots$ & $\mathrm{DnC}$ & 0.047 & $\ldots$ & $<0.152$ & $<0.318$ & 0.876 \\
\hline $211552.88+000115.4^{\mathrm{b}}$ & $\ldots$ & $\mathrm{D}$ & 0.041 & 0.32 & 0.103 & $\ldots$ & $>1.192$ \\
\hline $212019.13-075638.3$ & $\ldots$ & $\mathrm{D}$ & 0.041 & $\ldots$ & $<0.065$ & $<0.242$ & $>1.168$ \\
\hline $224749.57+134248.1$ & $1.175 ?$ & $\mathrm{D}$ & 0.041 & 0.98 & 0.088 & $\ldots$ & $>1.293$ \\
\hline $231000.83-000516.2$ & $\ldots$ & $\mathrm{D}$ & 0.040 & $\ldots$ & $<0.035$ & $<0.192$ & $>1.254$ \\
\hline $232428.43+144324.3$ & 1.410 & $\mathrm{D}$ & 0.048 & $\ldots$ & $<0.011$ & $\ldots$ & $>0.844$ \\
\hline
\end{tabular}

Notes.

${ }^{\text {a }}$ Configuration of the VLA during observations. D-array data were taken on 2007 March 30 as VLA program AP524; DnC-array data were taken on 2008 June 8 as VLA program AP551.

${ }^{\mathrm{b}}$ Might be a blend of two radio sources, both $\sim 1^{\prime \prime}$ from the optical position.

on 2008 June 8 for the other 12 . All observations were performed in the $\mathrm{X}$ band $(8.4 \mathrm{GHz})$ with 26 antennas, with a total of $20-30$ minutes integration on each source (using $10 \mathrm{~s}$ integrations). We used $2 \times 50 \mathrm{MHz}$ intermediate frequencies (IFs) centered at $v=8435 \mathrm{MHz}$ and $8485 \mathrm{MHz}$. Phase calibrators were observed every 10 minutes, and flux calibrations were tied to either $3 \mathrm{C} 48$ or 3C 286. A summary of the observations is given in Table 2. We observed at $8.4 \mathrm{GHz}$ because many BL Lac objects are brighter at higher radio frequencies (i.e., $\alpha_{r} \sim-0.27$ typically; Stickel et al. 1991). This combined with the VLA X band's superior sensitivity compared to other observing bands makes seeking radio detections for BL Lac candidates most efficient at 8.4 GHz.

We reduced the data using standard routines in AIPS, flagging anomalous $U V$ data when necessary. We cleaned the data and created maps with the IMAGR task, using a restoring beam $\sim 8^{\prime \prime}$ and $2^{\prime \prime} \times 2^{\prime \prime}$ pixels. We estimate background rms noises with TVSTAT, which range from $\sigma_{\text {rms }}=0.032$ from $0.048 \mathrm{mJy}_{\text {beam }}{ }^{-1}$. We require radio detections to have $8.4 \mathrm{GHz}$ flux densities $>5 \sigma_{\text {rms }}$ within $2^{\prime \prime}$ of the SDSS source position. We detect $4 / 17$ sources in the radio, and we measure their flux densities with TVSTAT using apertures 8-10 pixels on a side. The largest separation between the radio and SDSS optical positions for these four radio detections is 1 1"4.

We use the $8.4 \mathrm{GHz}$ flux densities (or limits) combined with SDSS optical fluxes to determine $\alpha_{\mathrm{ro}}^{\text {vla }}$, which is again calculated between rest frames $5 \mathrm{GHz}$ and $5000 \AA$. We follow the same methodology as in Section 2, placing $5 \sigma_{\text {rms }}$ upper limits on the radio flux densities for the non-detections. A conservative $5 \sigma_{\text {rms }}$ threshold is used for consistency with upper limits placed by the FIRST survey, because an extraordinary claim of a radio-quiet BL Lac discovery requires very stringent constraints. We include the values (or limits) of the radio-optical broadband spectral indices derived from our deeper VLA observations $\left(\alpha_{\mathrm{ro}}^{\text {vla }}\right)$ in Table 2, and we also include the broadband spectral indices derived from FIRST-SDSS $\left(\alpha_{\mathrm{ro}}^{\text {first }}\right)$ and RASS-SDSS $\left(\alpha_{\mathrm{ox}}^{\text {rass }}\right)$ for reference.
We find only a single object (SDSS J0241+0009) to be radioloud: it has an $8.4 \mathrm{GHz}$ flux density of $2.44 \mathrm{mJy}$, and we calculate $\alpha_{\mathrm{ro}}^{\text {vla }}=0.318$. This object also appears in FIRST with a $1.4 \mathrm{GHz}$ flux density of $0.67 \mathrm{mJy}$ (below the nominal FIRST flux limit) and $\alpha_{\mathrm{ro}}^{\text {first }}=0.249$. This source did not appear in the FIRST catalog at the times $\mathrm{C} 05$ and A07 were published, which is why we included it in our VLA program. The discrepancy between $\alpha_{\mathrm{ro}}^{\text {first }}$ and $\alpha_{\mathrm{ro}}^{\text {vla }}$ is primarily due to our choice of local radio spectral index $\alpha_{r}=-0.27$. Combining our 1.4 and $8.4 \mathrm{GHz}$ flux density measurements, we find $\alpha_{r}=-0.7$. If we adopt $\alpha_{r}=-0.7$, then $\alpha_{\mathrm{ro}}^{\text {first }}=0.273$ and $\alpha_{\mathrm{ro}}^{\text {vla }}=0.277$. Regardless, SDSS J0241+0009 is definitively radio-loud. It also has a flat radio spectrum, it is detected in the X-rays by RASS, and we show in Section 3.3 that it is variable. Although it is on the relatively radio-weak tail of the radio-loud BL Lac distribution, its radio-loudness is not extraordinarily small. It should therefore be considered a normal radio-loud BL Lac object.

The other three VLA radio detections have $\alpha_{\mathrm{ro}}^{\text {vla }}$ values that firmly place them in the radio-quiet regime. We note that one source (SDSS J2115+0001) might actually be a blend of two radio point sources (it is not clear with the spatial resolution of our observations). Nevertheless, we consider this source as a radio detection, and we report the total combined flux of the (potentially) two components. Even the combined radio flux confirms that SDSS J2115+0001 is radio quiet.

We conclude from the radio observations that the majority of weak-featured radio-quiet objects are indeed atypical in their radio properties from the much larger SDSS radio-loud BL Lac population. Of the 17 objects with deeper VLA coverage, we only classify a single source as a confident radio-loud BL Lac object (SDSS J0241+0009).

\subsubsection{VLA Observations of High-redshift WLQs}

Four $z>2.2$ WLQs included in C05's list of potential radioquiet BL Lac candidates were included in our VLA programs, and none was detected in the radio. We list their $8.4 \mathrm{GHz}$ radio properties in Table 3 for completeness. Of these four objects, 
Table 3

VLA Observations at $8.4 \mathrm{GHz}$ of High-redshift WLQs

\begin{tabular}{|c|c|c|c|c|c|c|c|}
\hline $\begin{array}{l}\text { SDSS Name } \\
(\mathrm{J} 2000)\end{array}$ & Redshift & Config $^{\mathrm{a}}$ & $\begin{array}{c}\sigma_{\mathrm{rms}} \\
\left(\mathrm{mJy} \mathrm{beam}^{-1}\right)\end{array}$ & $\begin{array}{c}f_{\text {vla }} \\
(\mathrm{mJy})\end{array}$ & $\alpha_{\mathrm{ro}}^{\mathrm{vla}}$ & $\alpha_{\mathrm{ro}}^{\text {first }}$ & $\alpha_{\mathrm{ox}}$ \\
\hline $031712.23-075850.3$ & 2.699 & $\mathrm{DnC}$ & 0.035 & $\ldots$ & $<-0.075$ & $\ldots$ & $1.705^{\mathrm{b}}$ \\
\hline $121221.56+534127.9$ & 3.190 & $\mathrm{DnC}$ & 0.038 & $\ldots$ & $<-0.108$ & $<0.072$ & $2.095^{\mathrm{b}}$ \\
\hline $123743.09+630144.9$ & 3.535 & $\mathrm{DnC}$ & 0.044 & & $<-0.113$ & $<0.058$ & $>1.671^{\mathrm{c}}$ \\
\hline $142505.59+035336.2$ & $2.248 ?$ & $\mathrm{DnC}$ & 0.038 & $\ldots$ & $<-0.058$ & $<0.126$ & $>1.236^{\mathrm{d}}$ \\
\hline
\end{tabular}

Notes.

${ }^{\text {a }}$ Configuration of the VLA during observations. Data were taken on 2008 June 8 as VLA program AP551.

${ }^{\mathrm{b}} \alpha_{\mathrm{ox}}$ value taken from Chandra detection in Shemmer et al. (2009), with their reported $\alpha_{\mathrm{ox}}$ value converted to conform to our $\alpha_{\mathrm{ox}}$ definition (see footnote 12).

${ }^{\mathrm{c}} \alpha_{\mathrm{ox}}$ limit taken from XMM-Newton non-detection in Shemmer et al. (2009), with their reported $\alpha_{\mathrm{ox}}$ limit converted to conform to our $\alpha_{\mathrm{ox}}$ definition (see footnote 12).

${ }^{\mathrm{d}} \alpha_{\mathrm{ox}}$ limit taken from RASS non-detection.

Table 4

Chandra Observations

\begin{tabular}{|c|c|c|c|c|c|c|c|c|}
\hline $\begin{array}{l}\text { SDSS Name } \\
(\text { J2000) }\end{array}$ & Redshift & $\begin{array}{l}\text { Exp. Time } \\
\text { (ks) }\end{array}$ & $\begin{array}{c}N_{H}^{\mathrm{a}} \\
\left(\mathrm{cm}^{-2}\right) \\
\end{array}$ & $\begin{array}{l}\text { Count Rate } \\
\left(\text { counts s }^{-1}\right)\end{array}$ & $\begin{array}{c}f_{X}^{\mathrm{c}} \\
\left(\mathrm{erg} \mathrm{s}^{-1} \mathrm{~cm}^{-2}\right)\end{array}$ & $\alpha_{\mathrm{ox}}^{\text {chandra }}$ & $\alpha_{\mathrm{ro}}^{\mathrm{vla}}$ & $\alpha_{\mathrm{ox}}^{\mathrm{rass}}$ \\
\hline $211552.88+000115.4^{\mathrm{d}}$ & & 9.5 & $5.83 \times 10^{20}$ & $<5 \times 10^{-4}$ & $<3.2 \times 10^{-15}$ & $>1.905$ & 0.103 & $>1.192$ \\
\hline $224749.57+134248.1^{\mathrm{e}}$ & $1.175 ?$ & 3.3 & $4.58 \times 10^{20}$ & $91_{-15}^{+21} \times 10^{-4}$ & $53.4 \times 10^{-15}$ & 1.552 & 0.088 & $>1.293$ \\
\hline $232428.43+144324.3^{\mathrm{f}}$ & 1.410 & 5.0 & $4.15 \times 10^{20}$ & $12_{-4}^{+8} \times 10^{-4}$ & $7.0 \times 10^{-15}$ & 1.796 & $<0.011$ & $>0.844$ \\
\hline
\end{tabular}

Notes.

${ }^{\text {a }}$ Column density from Stark et al. (1992).

${ }^{\mathrm{b}}$ From 0.5 to $6.0 \mathrm{keV}$, assuming a photon power law index $\Gamma=2.25$.

${ }^{\mathrm{c}}$ Corrected for Galactic absorption.

d Observation taken on 2008 December 24; Chandra ObsID 10388.

e Observation taken on 2009 August 7; Chandra ObsID 10387.

${ }^{\mathrm{f}}$ Observation taken on 2009 May 31; Chandra ObsID 10386.

three have deeper X-ray observations in Shemmer et al. (2009), and we include those objects' $\alpha_{\mathrm{ox}}$ measures derived from their deeper X-ray observations. ${ }^{12}$

\subsection{X-ray Observations with Chandra}

Of the 17 radio-quiet BL Lac candidates with deeper VLA observations, 14 only have lower limits on $\alpha_{\text {ox }}$ from RASS. Their RASS limits are not sensitive enough to determine if they are dissimilar to BL Lac objects in the X-ray, and deeper X-ray observations are required to compare their X-ray properties to the larger radio-loud BL Lac population.

We observed 3 of these 14 objects in the X-ray with Chandra during Cycle 10. The Chandra targets were chosen as the optically brightest of the 5 sources with VLA observations taken in 2007 (which were the only VLA observations we had in hand at that time). Chandra data were taken with the Advanced CCD Imaging Spectrometer (ACIS; Garmire et al. 2003) at the nominal S3 aimpoint, using faint telemetry mode. Exposure times of $9.5 \mathrm{ks}, 3.3 \mathrm{ks}$, and $5.0 \mathrm{ks}$ were achieved for SDSS J2115+0001, SDSS J2247+1342, and SDSS J2324+1443, respectively. The data were reduced using standard routines in CIAO ${ }^{13}$ The Chandra observations are summarized in Table 4.

\footnotetext{
12 Shemmer et al. (2009) use slightly different parameters for calculating $\alpha_{\mathrm{ro}}$ and $\alpha_{\text {ox }}$. Among the differences, their broadband spectral indices are defined with opposite sign compared to ours, and they are referenced at $2500 \AA$ in the optical and $2 \mathrm{keV}$ in the X-ray. They also assume local spectral indices of 0.5 in both the radio and the optical, and they use $\alpha_{v}=1.0$ in the X-ray. We convert their broadband spectral indices, $\alpha_{\mathrm{ro}}^{\prime}$ and $\alpha_{\mathrm{ox}}^{\prime}$, to be consistent with our adopted reference frequencies and local spectral indices using approximate transformations $\alpha_{\mathrm{ro}}=-1.059 \alpha_{\mathrm{ro}}^{\prime}+0.054$ and $\alpha_{\mathrm{ox}}=-1.116 \alpha_{\mathrm{ox}}^{\prime}-0.058$.

${ }^{13}$ Chandra Interactive Analysis of Observations. See http://cxc.harvard.edu/ciao/.
}

We filter the X-ray observations from 0.5 to $6.0 \mathrm{keV}$ to reduce the background. We then measure the number of Xray counts within 6 pixel circular apertures centered on each source's position with the CIAO routine dmextract. The 6 pixel apertures include $>90 \%$ of the encircled energy at the S3 aimpoint. ${ }^{14}$ The number of background counts is estimated over circular annuli with inner and outer radii of 10 and 20 pixels, respectively. No photons are detected for SDSS J2115+0001; we place an upper limit of 5 photons (99\% confidence interval, see Gehrels 1986) for SDSS J2115+0001, and we estimate a count rate $<0.0005$ counts $\mathrm{s}^{-1}$. SDSS $\mathrm{J} 2247+1342$ is detected with $30_{-5}^{+7}$ photons $\left(0.0091_{-0.0015}^{+0.0021}\right.$ counts s $\left.{ }^{-1}\right)$; a weak detection is obtained for SDSS J2324+1443, with $6_{-2}^{+4}$ photons $\left(0.0012_{-0.0004}^{+0.0008}\right.$ counts s $\left.{ }^{-1}\right) .{ }^{15}$

$\mathrm{X}$-ray fluxes from 0.5 to $6.0 \mathrm{keV}$ are estimated from the above count rates using PIMMS, correcting for Galactic absorption with the Stark et al. (1992) H I maps, and assuming an X-ray spectral index $\alpha_{\nu}=1.25$. We also estimate X-ray flux densities at $1 \mathrm{keV}$. Using the SDSS filter closest to rest frame $5000 \AA$, we find $\alpha_{\mathrm{Ox}}^{\text {chandra }}>1.90, \alpha_{\mathrm{OX}}^{\text {chandra }}=1.55$, and $\alpha_{\mathrm{ox}}^{\text {chandra }}=1.80$ for SDSS J2115+0001, SDSS J2247+1342, and SDSS J2324+1443, respectively.

The broadband spectral indices derived from Chandra for these 3 objects place them on the very X-ray faint tail of the radio-loud $\mathrm{BL}$ Lac $\alpha_{\mathrm{ox}}$ distribution. Among the 637 radio-loud optically selected BL Lac objects in P10, 294 are detected

\footnotetext{
${ }^{14}$ See the Chandra Proposers' Observatory Guide http://cxc.harvard.edu/proposer/POG/.

15 Quoted errors are $84.13 \%$ one-sided upper and lower confidence limits from Gehrels (1986). These are calculated using Poisson statistics, and they correspond to Gaussian $\pm 1 \sigma$ confidence intervals.
} 

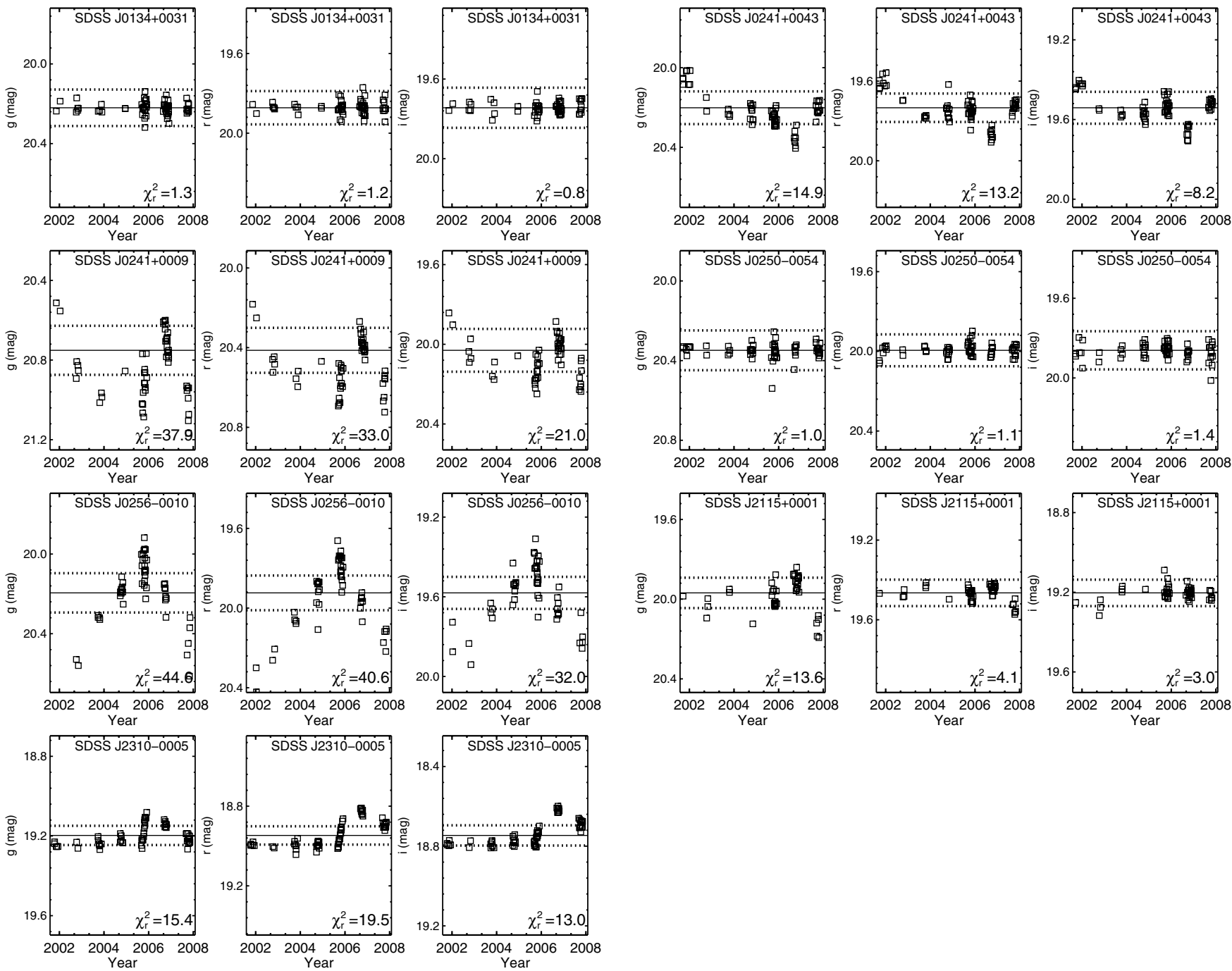

Figure 1. Stripe 82 light curves in the $g, r$, and $i$ filters for seven radio-quiet BL Lac candidates; data taken prior to Fall 2001 are not shown. The solid lines illustrate the average magnitude of each source, and the dotted lines mark \pm 3 times the average photometric error. All panels span 1 mag along the $y$-axis. The two non-variable objects (with $\chi_{r}^{2}<3$ ) are likely stars.

in the X-ray by RASS, with $\left\langle\alpha_{\text {ox }}\right\rangle=1.11 \pm 0.21$ (note, this implicitly accounts for variability). The largest $\alpha_{\mathrm{ox}}^{\text {rass }}$ for an object with an RASS X-ray detection is 1.66; only two BL Lac objects lacking RASS X-ray detections have larger $\alpha_{\mathrm{oX}}^{\text {rass }}$ limits. If these three objects are typical BL Lac objects with abnormally weak radio emission, then we might have expected their spectral energy distributions to peak at extremely high frequencies (i.e., they would be extreme high-energy-peaked BL Lac objects; see Padovani \& Giommi 1995a). However, in this scenario we would expect them to be relatively X-ray bright, which is excluded by our Chandra observations. We thus conclude that the objects with deeper Chandra observations are dissimilar to BL Lac objects in their X-ray properties, but the multiwavelength properties of these three objects are comparable to high-redshift SDSS WLQs (see Section 5.1).

Finally, we calculate $\Delta \alpha_{\text {ox }}$, the difference between the measured $\alpha_{\mathrm{ox}}^{\text {chandra }}$ values and that expected from their optical luminosities given the empirical $\alpha_{\mathrm{ox}}-L_{v}(2500 \AA)$ relation in Equation (3) of Just et al. (2007). $L_{v}(2500 \AA)$ is the specific luminosity at $2500 \AA$ rest frame, and for our $\Delta \alpha_{\text {ox }}$ measurements we re-calculate $\alpha_{\mathrm{ox}}^{\text {chandra }}$ at rest frames $2 \mathrm{keV}$ and $2500 \AA$ for consistency with Just et al. (2007). We find $\Delta \alpha_{\text {ox }}=$ $-0.052(-0.26 \sigma)$ and $0.197(0.994 \sigma)$ for SDSS $2247+1342$ and
SDSS J2324+1443, respectively. The values in parentheses are normalized to the standard deviation in $\alpha_{\mathrm{ox}}, \sigma=0.198$, for AGN with optical luminosity densities between $10^{30}$ and $10^{31} \mathrm{erg} \mathrm{s}^{-1}$ $\mathrm{Hz}^{-1}$ (see Table 5 of Steffen et al. 2006). The lack of a spectroscopic redshift (or an X-ray detection) for SDSS J2115+0001 precludes us from estimating its $\Delta \alpha_{\mathrm{ox}}$ value. The measured $\Delta \alpha_{\mathrm{ox}}$ for SDSS 2247+1342 and SDSS J2324+1443 is not significantly different from zero, and we thus conclude that their X-ray properties are similar to normal radio-quiet quasars and WLQs (see Shemmer et al. 2009).

\subsection{Optical Flux Variability}

Finally, we consider flux variability, as we expect strong variability if these are in fact BL Lac objects. The SDSS has observed a $\sim 300 \mathrm{deg}^{2}$ region of the sky $\left(20^{\mathrm{h}} 34^{\mathrm{m}}<\alpha_{\mathrm{J} 2000.0}<\right.$ $\left.04^{\mathrm{h}} 00^{\mathrm{m}},\left|\delta_{\mathrm{J} 2000.0}\right|<1.266\right)$ in all five SDSS filters multiple times every Fall since 1998. This region is referred to as Stripe 82 (see Ivezić et al. 2007; Sesar et al. 2007). Seven of our lowredshift radio-quiet BL Lac candidates lie inside Stripe 82, each one with 50-70 epochs of observations (see Figure 1). The $u$ and $z$ filters tend to be noisier, so we do not consider them here. Most objects only have 1-2 epochs of observations per year 
Table 5

Optical Variability of the Seven Objects in Stripe 82

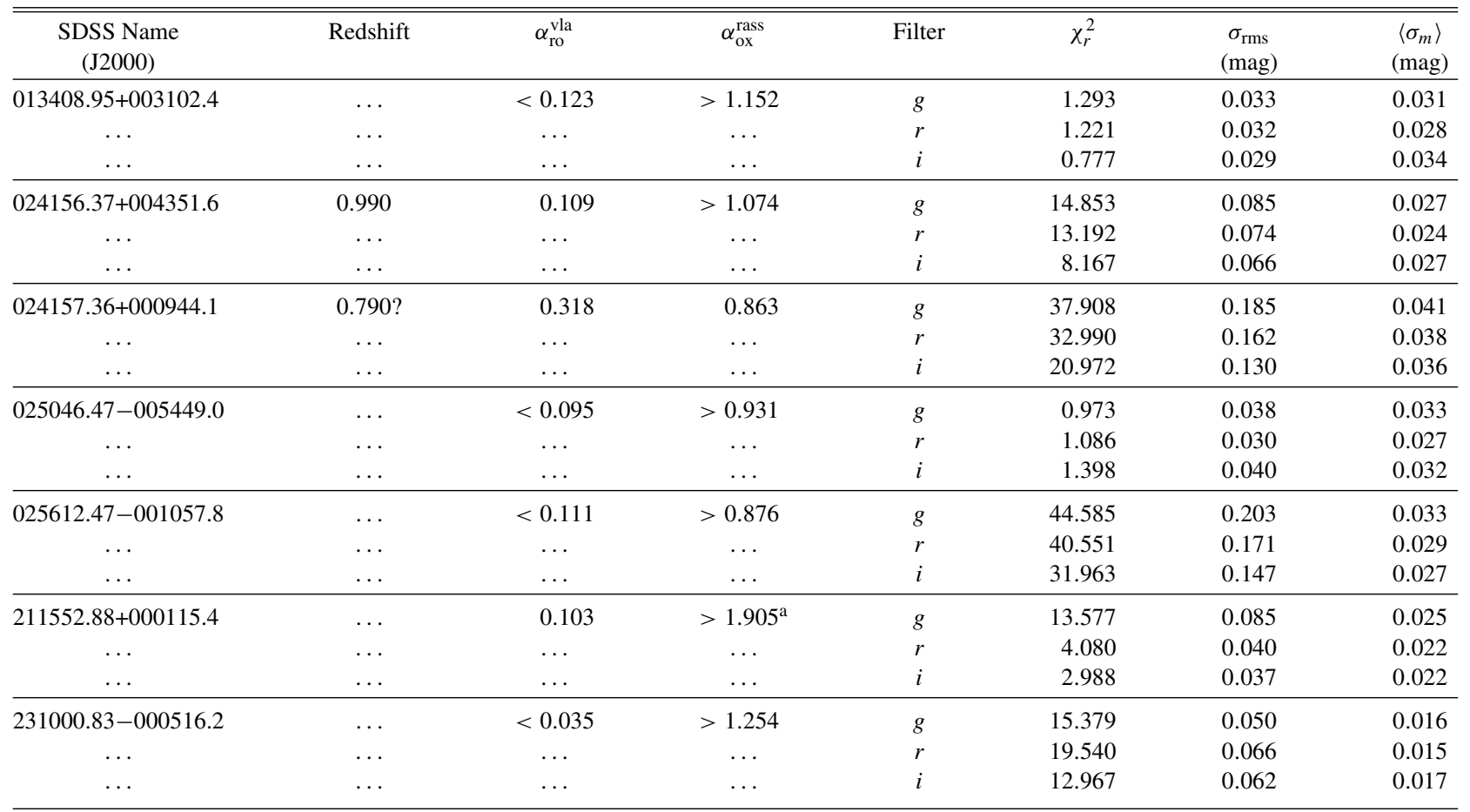

Note. ${ }^{a}$ X-ray flux limit from Chandra.

prior to Fall 2001, so we do not show these data in Figure 1, but we do include pre-2001 epochs in the following analysis.

We use the reduced $\chi_{r}^{2}$ statistic to assess the level of flux variability in a given filter. ${ }^{16}$ If an object has $\chi_{r}^{2}>3$, then we consider it to show variability (e.g., see Sesar et al. 2007). We find five of the seven sources to be variable in the $g$ filter (SDSS J0241+0043, SDSS J0241+0009, SDSS J0256-0010, SDSS J2115+0001, and SDSS J2310-0005). These five objects show flux variations over short and long timescales (ranging from days to years), and flux variations tend to be correlated between filters. We note that one of the variable objects is SDSS J0241+0009, which we classify as a radio-loud BL Lac object based on its radio and X-ray properties.

The measured values of $\chi_{r}^{2}$ and the rms scatter, $\sigma_{\text {rms }}$, for each source in each filter are listed in Table 5. We include $\alpha_{\text {ro }}^{\text {vla }}$ and $\alpha_{\mathrm{ox}}^{\text {rass }}$ for reference, and we list the average measured photometric error for each source in each filter, $\left\langle\sigma_{m}\right\rangle$. Observations typically have photometric uncertainties of $\sim 0.03 \mathrm{mag}$, and the largest degree of variability we detect is $\sigma_{\text {rms }} \sim 0.2 \mathrm{mag}$ (SDSS J0256-0010). The two objects that do not appear variable are likely stars. Neither has a spectroscopic redshift, and one of them (SDSS J0134+0031) has a very large proper motion

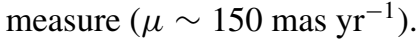

The detection of variability does not necessarily indicate that these are BL Lac objects, because radio-quiet quasars are also variable. We now compare the level of variability of the radio-quiet $\mathrm{BL}$ Lac candidates to normal radio-loud $\mathrm{BL}$ Lac objects and to radio-quiet quasars. There are 14 optically selected BL Lac candidates in P10 with Stripe 82 light curves that are detected in the radio by FIRST/NVSS and are radio-

$16 \chi_{r}^{2}=\sum_{i}^{N}\left[\left(m_{i}-\langle m\rangle\right) / \sigma_{i}\right]^{2} /(N-1)$, where $m_{i}$ and $\sigma_{i}$ are the observed magnitudes and uncertainties, respectively, in a given filter, $\langle m\rangle$ is the average magnitude, and $N$ is the number of epochs in that filter. loud; we calculate $\chi_{r}^{2}$ for those objects in the $g$-filter. ${ }^{17} \mathrm{We}$ also examine a sample of $\sim 2000$ spectroscopically confirmed radio-quiet SDSS DR5 quasars in Stripe 82 from Schneider et al. (2007), and we measure $\chi_{r}^{2}$ for each of these radio-quiet quasars. These $\sim 2000$ quasars are selected because (1) they have Stripe 82 light curves with at least 10 epochs of data in each filter; (2) they are in the FIRST survey's footprint and have $\alpha_{\text {ro }}<0.2$, derived from FIRST radio detections or limits; (3) they have $g>18.7$, so that their optical fluxes are similar to our radio-quiet BL Lac candidates with Stripe 82 coverage; and (4) they have $0.5<z<2.2$. This redshift range encompasses the redshifts of our seven radio-quiet BL Lac candidates in Stripe 82. All have $z<2.2$, since we do not see the $\operatorname{Ly} \alpha$ forest in their SDSS spectra; those lacking spectroscopic redshifts probably have $z>0.5$, or else we would likely see host galaxy spectral features in their optical spectra (see P10). We restrict our comparison quasar sample to these redshifts so that our $\chi_{r}^{2}$ measures are calculated at approximately similar rest frames. For simplicity, the following analysis is illustrated with values derived in the $g$ filter, but our conclusions do not change if we use a different filter (or if we choose filters to cover similar rest-frame wavelengths).

Figure 2 shows $\chi_{r}^{2}$ versus $\alpha_{\text {ro }}$ for the six radio-quiet BL Lac candidates with Stripe 82 light curves (squares, not including SDSS J0241+0009) and for the 14 radio-loud BL Lac candidates (circles) from P10 that lie in Stripe 82. We use VLA data when available to calculate $\alpha_{\text {ro }}$. The $\sim 2000$ radio-quiet quasars have an average $\left\langle\chi_{r}^{2}\right\rangle=65$, and $84 \%$ (i.e., the one-sided $1 \sigma$

\footnotetext{
17 Because SDSS J0241+0009 has radio-loud $\alpha_{\text {ro }}$ measures from both FIRST and our follow-up VLA observations, we do not consider it a radio-quiet BL Lac candidate in the following. It was recovered as a radio-loud BL Lac candidate in P10, and it is thus included in the 14 object subset of radio-loud BL Lac objects with Stripe 82 coverage.
} 


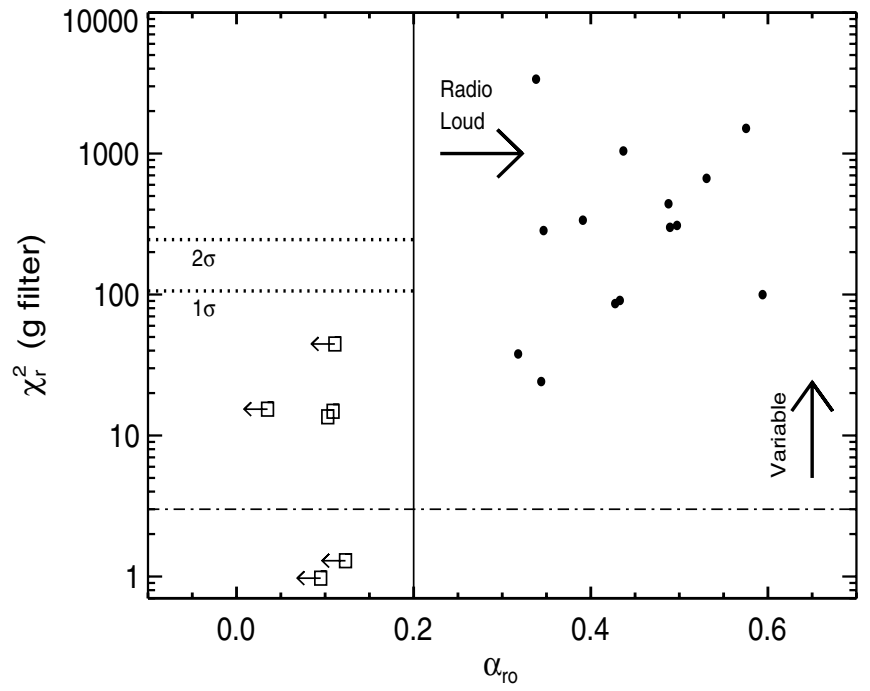

Figure 2. Reduced $\chi_{r}^{2}$ of flux variability in the $g$ filter vs. $\alpha_{\text {ro }}$. Circles denote 14 radio-loud BL Lac objects from P10 with Stripe 82 light curves. Squares denote 6 radio-quiet BL Lac candidates with Stripe 82 light curves and $\alpha_{\text {ro }}$ measures (or limits) smaller than 0.2 . The vertical solid line shows the traditional boundary between radio-loud and radio-quiet AGNs. The horizontal dash-dotted line shows $\chi_{r}^{2}=3$, above which we conclude a source is variable. The dotted lines mark the maximum values of $\chi_{r}^{2}$ we expect $84 \%(1 \sigma)$ and $97.5 \%(2 \sigma)$ of radio-quiet quasars to show. The variability of the radio-quiet BL Lac candidates is consistent with that expected for radio-quiet quasars, although there is some overlap between the high and low variability tails of the radio-quiet quasar and BL Lac distributions. This figure is similar if we rather plotted $\chi_{r}^{2}$ for the $r$ or $i$ filters.

confidence interval) have $\chi_{r}^{2}<106$, and $97.5 \%$ (i.e., the onesided $2 \sigma$ confidence interval) have $\chi_{r}^{2}<245$ (marked by the dotted lines in Figure 2). ${ }^{18}$ There is a marked deficit of extremely variable radio-quiet objects.

The level of flux variability is likely dominated by different physical processes in radio-quiet and radio-loud quasars. Using a sample of $\sim 100$ quasars covering a wide range of redshifts, optical luminosities, and radio loudnesses, Kelly et al. (2009) suggest the flux variability of radio-quiet quasars is caused by a stochastic process; this conclusion is confirmed by MacLeod et al. (2010). One potential mechanism proposed by Kelly et al. (2009) to explain the observed variability for radio-quiet quasars is turbulent magnetic fields within the accretion disk. For radioloud quasars, however, additional mechanisms related to the radio jet need to be invoked. Here, variability is commonly modeled as shocks propagating through an inhomogeneous jet (e.g., see Marscher \& Gear 1985; Ulrich et al. 1997; Aller et al. 1999).

The $\chi_{r}^{2}$ values in Figure 2 suggest that the mechanism driving variability in the radio-quiet BL Lac candidates is similar to that for radio-quiet quasars. To test this assertion, we assume the observed distribution of $\chi_{r}^{2}$ for the four variable radio-quiet $\mathrm{BL}$ Lac candidates is randomly drawn from the $\chi_{r}^{2}$ distribution of the $\sim 2000$ DR5 radio-quiet quasars. We ignore the two nonvariable radio-quiet BL Lac candidates because they are likely stellar. We also assume that the 14 radio-loud BL Lac objects' $\chi_{r}^{2}$ measurements are randomly drawn from the DR5 quasars. The above null hypotheses are then tested via Monte Carlo simulations, as described below. This test is designed to achieve the same purpose as a Kolmogorov-Smirnov test, but we use Monte Carlo simulations because of the small sample sizes.

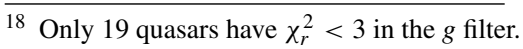

Of the 14 P10 radio-loud BL Lac objects with Stripe 82 light curves, $12 / 14$ have $\chi_{r}^{2}>65$ (the mean of the DR5 quasars), $9 / 14$ have $\chi_{r}^{2}>106(1 \sigma)$, and 9/14 have $\chi_{r}^{2}>245(2 \sigma)$. To test the likelihood that the $\sim 2000$ DR5 quasars are the parent population of the 14 radio-loud BL Lac objects, we randomly choose $14 \chi_{r}^{2}$ values from the DR5 quasar distribution. We then count the number of randomly selected $\chi_{r}^{2}$ values larger than 65 , 106, and 245; we repeat $10^{6}$ times. Our Monte Carlo simulations show there are $p=0.000091,0.000061$, and $<0.000001$ chances of randomly drawing 12,9 , and 9 objects with $\chi_{r}^{2}$ values larger than 65,106 , and 245 , respectively. We thus conclude that the radio-loud BL Lac objects are statistically different from the DR5 quasars in their variability properties.

For the four radio-quiet BL Lac candidates, no object has measured $\chi_{r}^{2}>65$. We perform a similar test, this time randomly drawing only four $\chi_{r}^{2}$ values from the distribution of DR5 quasars, and we again repeat $10^{6}$ times. We find that there are $p=0.19,0.50$, and 0.90 chances of randomly choosing zero quasars with $\chi_{r}^{2}>65,106$, and 245 , respectively. Thus, we conclude it is plausible the level of variability displayed by our radio-quiet BL Lac candidates is similar to that of normal radio-quiet quasars. We of course caution that one should not draw strong statistical conclusions from these small sample sizes; however, our Monte Carlo simulations suggest that the radio-quiet BL Lac candidates are similar to radioquiet quasars, but the radio-loud BL Lac objects are not. This conclusion is strengthened after also considering their radio and $\mathrm{X}$-ray properties. It is also important to mention that there is overlap in $\chi_{r}^{2}$ between the most variable radio-quiet quasars and the least variable radio-loud BL Lac objects. Thus, we cannot exclude that some of the more variable radio-quiet $\mathrm{BL}$ Lac candidates (e.g., SDSS J0256-0010) have weakly boosted continua, but that does not appear to be the dominant source of radiation. A similar conclusion was reached for some relatively radio-bright high-redshift WLQs (e.g., SDSS J1408+0205 and SDSS J1442+0110; Diamond-Stanic et al. 2009). The most variable radio-quiet BL Lac candidates should be placed at high priority for polarimetric follow-up.

\section{RECOGNITION OF SOURCES UNLIKELY TO BE LINELESS AGN}

Equipped with the above observations, we now identify objects that are unlikely to be AGN with intrinsically weak emission lines. Here we discuss all 26 radio-quiet BL Lac candidates in Table 1, not just the 17 with deeper VLA observations. Improvements to the SDSS data reduction pipelines are implemented prior to each data release, so we also inspected the DR7 spectra of the C05 and A07 radio-quiet BL Lac candidates (which were selected from earlier SDSS data releases).

\subsection{Normal Radio-loud BL Lac Object}

SDSS J0241+0009 is a normal radio-loud BL Lac object based on its radio and X-ray properties, and its optical variability.

\subsection{Stars}

Three of the twenty-six radio-quiet BL Lac candidates show large proper motion in Table 1 , which we define as $\mu>$ $30 \mathrm{mas} \mathrm{yr}^{-1}$ (less than $1 \%$ of spectroscopically confirmed SDSS quasars have proper motion measures larger than 30 mas $\mathrm{yr}^{-1}$; Schneider et al. 2007). One of these measurements is spurious: SDSS J0241+0009 $\left(\mu=54 \mathrm{mas} \mathrm{yr}^{-1}\right)$ is a very convincing radio-loud BL Lac object (see Section 4.1). The other two 
sources with large proper motion measures (SDSS J0134+0031

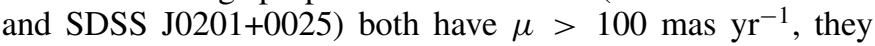
lack spectroscopic redshifts, and they have point source optical morphologies. ${ }^{19}$ Neither is detected by our deeper radio observations or by RASS in the X-ray. We have an optical light curve for SDSS J0134+0031 (see Section 3.3), and it does not show variability over an entire decade. Those two proper motion measures are likely not spurious, and those two objects are removed from the sample as potential stars. We also remove SDSS J0250-0054 as a likely star because it does not show flux variability in its Stripe 82 light curve, it does not have a measured redshift, and it is not detected in our $8.4 \mathrm{GHz}$ VLA observations. The spectrum of SDSS J1147+3513 might be contaminated by a bright nearby star, and SDSS J2120-0756 might also show weak stellar absorption features in its DR7 spectrum. We thus remove those two sources as well (neither has a measured redshift). In all, 5 of the 26 radio-quiet BL Lac candidates are removed because they are probably stars.

\subsection{Galaxies}

Examination of the improved SDSS spectral reductions of SDSS J1431+6006 and SDSS J2139+1047 show they have $\mathrm{Ca}$ II $\mathrm{H} / \mathrm{K}$ breaks larger than $40 \%$, and we re-classify them as elliptical galaxies. We also remove SDSS J1242+6429, which is probably a galaxy: its very low redshift $(z=0.042)$ and low optical luminosity $\left(\log v_{o} L_{v_{o}}<43 \mathrm{erg} \mathrm{s}^{-1}\right)$ suggest that it is not an AGN.

\subsection{Absorbed AGN}

The weak emission lines from SDSS J1511+5637 and SDSS J1658+6118 might be explained by absorption. Both objects have extremely red spectra (optical spectral indices $\alpha_{v}=4.4$ and 5.9, respectively), and SDSS J1658+6118 is additionally classified as a broad absorption line quasar in Trump et al. (2006).

\subsection{Misidentified Optical Counterparts to RASS X-ray Sources}

There are sometimes multiple optical sources within each 60 " RASS X-ray circle, and we believe that two radio-quiet BL Lac candidates (SDSS 0755+3525 and SDSS J1610+2536) are not the proper optical counterparts. SDSS $0755+3525$ is $48^{\prime \prime}$ from the center of the RASS error circle. There is a more likely optical counterpart (identified as a starburst galaxy by Brinkmann et al. 2000) just $3^{\prime \prime}$ away from the RASS source and 0 '. 2 from a FIRST radio source (FIRST J075551.3+352635). For SDSS J1610+2536, there is a bright star 16" away (cataloged as V1024 Her, an eclipsing variable star with $V=12.5 \mathrm{mag}$ in SIMBAD) that is also inside the RASS X-ray circle. V1024 Her is probably the X-ray emitter. Although its spectral type is not available in the literature (and it is too bright to have an SDSS spectrum), its optical magnitude and RASS X-ray count rate are consistent with those expected from a K or M star (see Figure 3 of Agüeros et al. 2009). We remove both sources from our list of radio-quiet BL Lac candidates.

\section{DISCUSSION}

We are left with 13 AGNs with weak emission features (see Figure 3), 10 of which are confirmed to be radio quiet from our VLA observations. The other three sources' FIRST radio limits

\footnotetext{
${ }^{19}$ Both objects are from $\mathrm{C} 05$, and their proper motions were less certain at the time of publication.
}

are not sensitive enough to determine if they are radio quiet. Our 13 surviving weak-featured AGNs are likely a heterogeneous combination of at least two populations of objects. Here, we classify them as likely low-redshift WLQs, as radio-faint BL Lac objects, or as uncertain. These classifications are listed in Table 1. We also compare them to other low- and high-redshift radio-quiet AGNs in the literature with weak or absent spectral features.

\subsection{Low-redshift WLQs}

The multiwavelength colors of our 13 radio-quiet BL Lac candidates are shown in Figure 4. The three objects with Chandra coverage (SDSS J2115+0001, SDSS J2247+1342, and SDSS J2324+1443, open upside down blue triangles) are radio and X-ray fainter than normal BL Lac objects. SDSS J2115+0001 does not have a redshift, but it does have an $8.4 \mathrm{GHz}$ radio detection implying $\alpha_{\text {ro }}=0.1$. It also shows weak variability in its Stripe 82 light curve. Smith et al. (2007) performed a polarimetric survey of $42 \mathrm{BL}$ Lac candidates from $\mathrm{C} 05$, and they did not detect strong polarization from either SDSS J2247+1342 or SDSS J2324+1443 (both have polarization $P<1 \%$ ). This further suggests they should not be unified with BL Lac objects. Based on their low polarization and multiwavelength colors, they may instead be low-redshift analogs to WLQs.

The prototype high-redshift WLQ was discovered by Fan et al. (1999; SDSS J1532-0039; $z=4.62$ ), who remarked on a "high-redshift quasar without emission lines." SDSS J15320039 was not detected in the X-ray with follow-on Chandra observations (Shemmer et al. 2006); it also remained undetected by the VLA (clearly placing it as radio quiet), and optical observations also did not find any polarization or strong variability (Diamond-Stanic et al. 2009). Anderson et al. (2001) discovered two more $z=4.5-4.6$ WLQs, and a fourth discovery at $z=5.9$ was reported by Fan et al. (2006). Diamond-Stanic et al. (2009) present a sample of $\sim 70$ SDSS WLQs $(z>3)$, defined by Ly $\alpha+\mathrm{N} v$ REW $<15 \AA$. They show that WLQs constitute a prominent excess of objects in the low $\mathrm{Ly} \alpha+\mathrm{N} v$ equivalent width tail of the high-redshift quasar distribution (which otherwise follows a log-normal distribution), and there is no corresponding excess of objects in the high-REW tail.

While the SDSS has produced a relatively large number of high-redshift discoveries, there are only a handful of low-redshift candidates in the literature. To our knowledge, PG 1407+265 ( $z=0.94$; McDowell et al. 1995) was the first AGN with weak emission features not interpreted as a BL Lac phenomenon. PG $1407+265$ has radio, X-ray, and optical flux ratios typical of normal radio-quiet quasars. McDowell et al. (1995) present UV, optical, and near-infrared spectra, and they find that all lines (in particular, $\mathrm{Ly} \alpha, \mathrm{C}$ IV, and $\mathrm{Mg}$ II) have unusually small equivalent widths. However, they do detect comparatively strong $\mathrm{H} \alpha$ by this odd source's standards (REW $=126 \AA$, which is still relatively weak compared to most quasars). Blundell et al. (2003) report the detection of a relativistically boosted core for PG 1407+265, which might (help) explain its odd spectral characteristics. Even with the detection of a radio core, Blundell et al. (2003) do not believe that PG 1407+265 has been radio-loud within the last $10^{6}-10^{7}$ years.

Leighly et al. (2007a) present a detailed multiwavelength spectral coverage of PHL $1811(z=0.192)$, an unusual X-ray weak narrow-line type 1 quasar. They detect relatively strong $\mathrm{H} \alpha$ and $\mathrm{H} \beta$ emission, but forbidden, semiforbidden, and high ionization lines are extraordinarily weak or absent. PHL 1811 


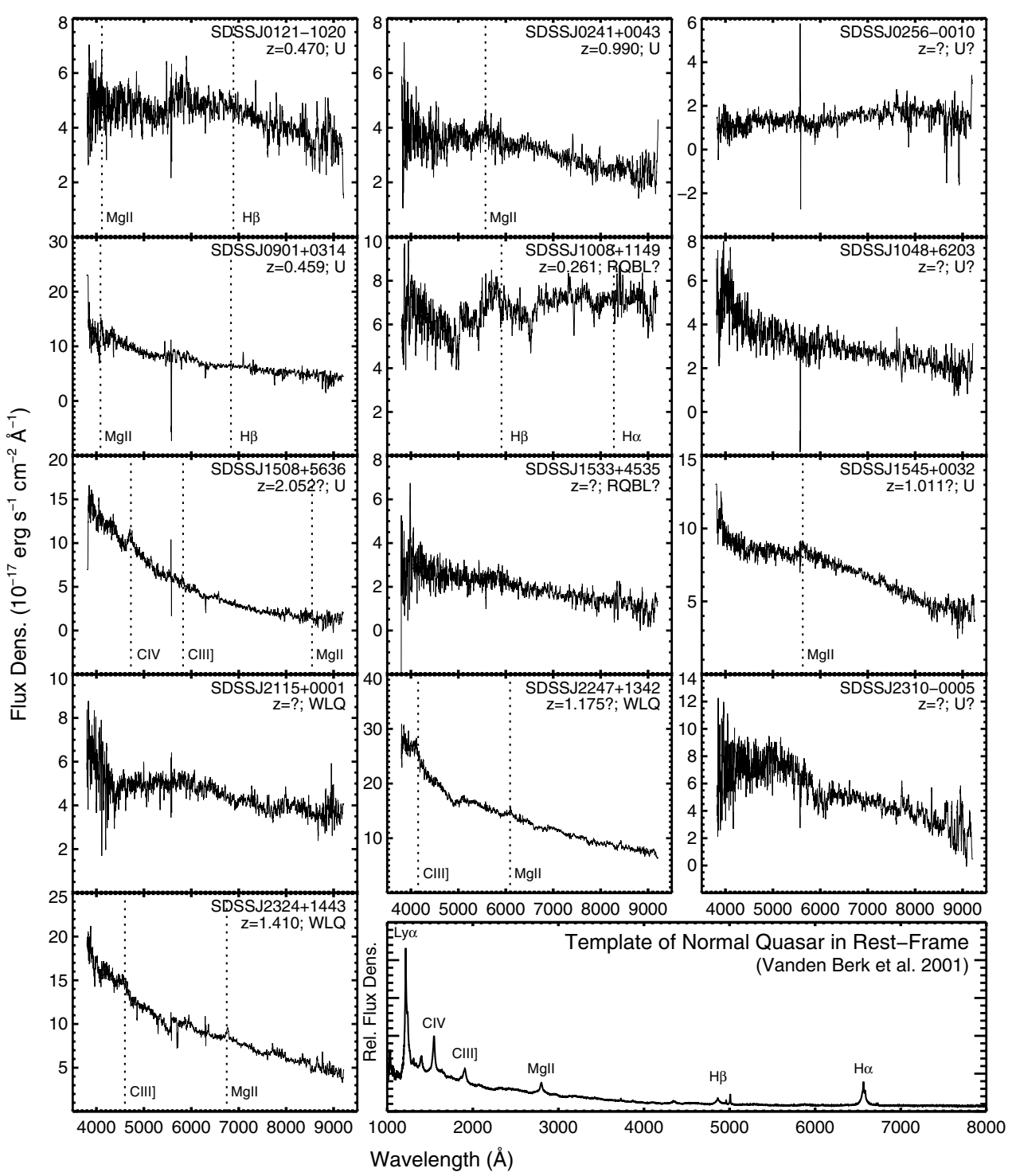

Figure 3. SDSS spectra of the 13 surviving weak-featured AGNs in the observed frame. In the bottom right panel we show, for reference, the Vanden Berk et al. (2001) composite SDSS quasar spectral template (shown in the rest-frame), with prominent broad emission lines labeled. The expected locations of these lines are drawn for the radio-quiet BL Lac candidates with redshifts. Redshifts smaller than 0.5 are derived from host galaxy absorption features (not labeled). Our classification as weak line quasar (WLQ), radio-quiet BL Lac candidate (RQBL?), or unknown (U) from Table 1 is given after each object's redshift. Some panels show a residual sky line at $5500 \AA$.

also shows relatively strong Fe II and Fe III emission. We note in particular that the SDSS spectra of SDSS J2247+1342 and SDSS J2324+1442 look similar to PHL 1811, and we might expect them to also have strong Fe II and Fe III lines.

Leighly et al. (2007a) run photoionization models and find that almost all of the odd spectral characteristics of PHL 1811 can be explained by its soft SED, as fewer high-ionization species are formed than would result from a harder (and more normal) SED. Most of the radiative cooling is performed via hydrogen lines (which can be excited by the soft continuum), hence the relatively strong low-order Balmer lines. Leighly et al. (2007b) show PHL 1811 has a steep X-ray photon spectral index $(\Gamma \sim 2.3$ over $0.3-5.0 \mathrm{keV})$, and they attribute this to an exceptionally high (perhaps super-Eddington) accretion rate. Shemmer et al. (2009) note that Chandra detected that highredshift SDSS WLQs do not appear to have unusually steep hard X-ray spectra, but they stress that X-ray observations with improved photon statistics are required to test this properly. Infrared spectroscopy to determine the strength of $\mathrm{H} \alpha$ and/or
$\mathrm{H} \beta$ for SDSS WLQs would also be illuminating, and $\mathrm{H} \beta$ line widths may allow accretion rate estimates (e.g., see Shemmer et al. 2004).

A proposed lower-redshift analog to high-redshift WLQs was serendipitously discovered in the SDSS spectroscopic database by Hryniewicz et al. (2010; SDSS J094533.99+100950.1, $z=$ 1.66). SDSS J0945+1009 has a normal optical continuum compared to radio-quiet quasars, but it shows very weak $\mathrm{Mg}$ II emission (REW $\sim 15 \AA$ ) and absent C III] and C IV emission. SDSS J0945+1009 is similar to the intermediate-redshift radioquiet BL Lac candidates presented in Table 1; however, even its weak Mg II is too large to make it into our SDSS BL Lac samples.

\subsection{Radio-quiet BL Lac Candidates}

Two objects (SDSS J1008+1149 and SDSS J1533+4535) are detected in the X-ray by RASS, with X-ray brightnesses typical of BL Lac objects $\left(\alpha_{\text {ox }}=0.99\right.$ and 1.22 , respectively; see Figure 4). Both objects appear to be the best optical 


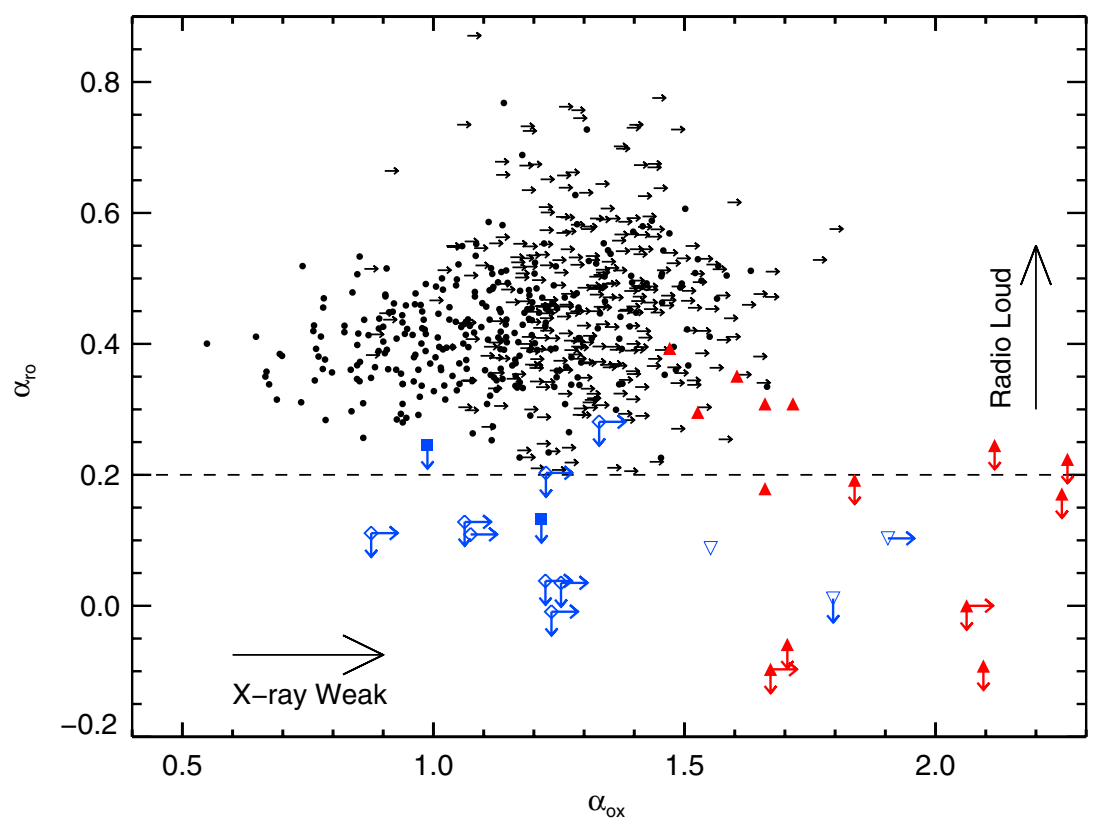

Figure 4. Broadband spectral indices of 13 surviving $z<2.2$ radio-quiet BL Lac candidates, with arrows denoting limits. Two objects with RASS X-ray detections retained (with low-confidence) are identified as potential very radio-faint BL Lac objects (filled blue squares). Eight objects lacking sensitive X-ray constraints are shown (open blue diamonds). Three objects with Chandra X-ray observations, which are likely low-redshift WLQs are shown (open upside-down blue triangles). For reference, we show 14 high-redshift $(2.7<z<5.9)$ WLQs (filled red triangles) with Chandra/XMM X-ray follow-up from Shemmer et al. (2006, 2009). Their radio information is typically derived from FIRST/NVSS, except for three WLQs with follow-up VLA observations in Table 3 . We also plot $\sim 600$ optically selected radio-loud BL Lac objects. Circles denote BL Lac objects with an X-ray detection in RASS and arrows mark X-ray limits for objects lacking RASS detections. The dashed line shows the traditional boundary between radio-loud/quiet quasars $\left(\alpha_{\mathrm{ro}}=0.2\right)$.

(A color version of this figure is available in the online journal.)

counterparts in each RASS error circle, but of course Chandra observations would be helpful for confirmation. These sources are very interesting, but we cannot currently assert that they are extraordinary radio-quiet BL Lac objects: we do not have deeper VLA constraints for SDSS $1008+1149$, and its FIRST flux limit $\left(\alpha_{\mathrm{ro}}<0.25\right)$ does not firmly identify it as radio quiet. Its redshift $(z=0.26)$ is also very typical of the parent SDSS BL Lac sample, and, similar to SDSS J0241+0009, this could simply be a relatively radio-faint but otherwise normal high-energy peaked BL Lac object. SDSS J1533+4535 was not detected in deeper VLA observations, and it is radio fainter than normal BL Lac objects at the $>3.4 \sigma$ level. However, its SDSS spectrum is relatively noisy. Both sources should be placed at high priority for further study.

\subsection{Unknown}

The remaining eight objects are very likely AGNs, but their RASS X-ray limits are not sensitive enough to determine if they are X-ray weaker than BL Lac objects (see Figure 4). Deeper $\mathrm{X}$-ray observations are necessary to determine if they are best unified with BL Lac objects or with WLQs. We only lack deeper VLA observations for two objects - SDSS J0901+0314 and SDSS J1048+6203.

Three objects (SDSS J0241+0043, SDSS J1508+5636, and SDSS J1545+0032) are particularly difficult to unify with BL Lac objects because they are at relatively high redshifts: they all have $z>1$, and the median redshift of radio-loud SDSS BL Lac objects from P10 with reliable redshifts is 0.34 . Polarimetric observations by Smith et al. (2007) also found SDSS J1545+0032 to not be highly polarized $(P \sim 1 \%)$. All three objects have $\alpha_{\mathrm{ro}}^{\text {vla }}<0.2$, and we suggest they are most likely lower-redshift WLQs (although we do so with low confidence given their poor X-ray constraints).
Two objects, SDSS J0121-1020 and SDSS J0901+0314, have redshifts $(z=0.47$ and $z=0.46$, respectively) typical of normal BL Lac objects. SDSS J0121-1020 is definitively radio quiet $\left(\alpha_{\text {ro }}<0.04,>4.75 \sigma\right.$ radio fainter than normal $\mathrm{BL}$ Lac objects); we do not have deeper VLA observations for SDSS J0901+0314, but its FIRST radio flux limit $\left(\alpha_{\text {ro }}^{\text {first }}<0.20\right)$ indicates that it is radio fainter than BL Lac objects at the $>2.75 \sigma$ level. Deeper X-ray observations should be taken for both sources to determine if they are more likely very low redshift WLQs or if they are very radio faint BL Lac objects.

Neither source shows obvious signatures of star formation or for obscured emission lines, and their $\mathrm{Ca}$ II $\mathrm{H} / \mathrm{K}$ depressions are much smaller than expected for normal elliptical galaxies. We do note that weak stellar absorption lines are seen in the spectrum of SDSS J0901+0314, which suggests it might be a post-starburst galaxy. However, an enhanced blue continuum from recent star formation alone is unlikely strong enough to explain its weak $\mathrm{Ca}$ II $\mathrm{H} / \mathrm{K}$ break and lack of strong emission lines. For example, SDSS J0901+0314 shows only very weak H $\delta$ with REW of $1-2 \AA$ in absorption, and post-starburst galaxies typically require $\mathrm{H} \delta$ stronger than $5 \AA$ in absorption (e.g., see Goto 2005).

Interestingly, these two objects appear remarkably similar to the radio-quiet BL Lac candidate 2QZ J215454.3-305654 ( $z=$ 0.49 ) discovered by Londish et al. (2004); 2QZ J2154-3056 shows only weak [O III] and perhaps some very weak Balmer emission. ${ }^{20}$ Both SDSS J0121-1020 and SDSS J0901+0314 also show weak forbidden lines from oxygen and very weak narrow $\mathrm{H} \beta$ (all lines have REW $<5 \AA$ ). Londish et al. (2004) do not detect optical polarization or radio emission $\left(\alpha_{\mathrm{ro}}<0.08\right)$,

\footnotetext{
${ }^{20} \mathrm{H} \alpha$ is not in the spectral coverage of their optical spectrum. Ca II $\mathrm{H} / \mathrm{K}$ and G-band absorption from the host galaxy are also detected.
} 
arguing against a beamed synchrotron continuum for $2 \mathrm{QZ}$ J2154-3056. X-ray emission is not seen in RASS $\left(f_{X}<10^{-14}\right.$ erg $\mathrm{s}^{-1} \mathrm{~cm}^{-2}$ ), and we estimate from their Figure 2 that $\alpha_{\text {ox }}>1.2$. They do detect marginal variability; the variability, however, is seen in only one of the four epochs over a $\sim 2-3$ month period with a reduced $\chi_{r}^{2}=4$. The lack of relatively strong $\mathrm{H} \beta$, and its redder spectrum, makes 2QZJ 2154-3056 dissimilar to PHL 1811 and PG 1407+265.

2QZJ 2154-3056 and the similar SDSS sources are unlikely explained by near- or super-Eddington accretion rates (as in PHL 1811), because then we would expect relatively strong $\mathrm{H} \beta$. It is alternatively possible to create lineless AGN with exceptionally low accretion rates $\left(\lesssim 10^{-2}\right.$ to $\left.10^{-3} \dot{M}_{\text {Edd }}\right)$, as has been invoked for the so-called naked AGN (i.e., Type 1 Seyfert galaxies lacking broad emission lines; Hawkins 2004) and X-ray bright, optically normal galaxies (XBONGS; e.g., see Trump et al. 2009). At these low accretion rates, the inner region of the accretion disk becomes radiatively inefficient. Thus, a radiation pressure driven wind never forms the BELR (e.g., see Nicastro 2000; Nicastro et al. 2003). Elitzur \& Ho (2009) reach a similar conclusion based on mass conservation arguments.

SDSS J0121-1020 and SDSS J0901+0314 are, however, unlikely to have low-enough accretion rates for their BELRs to vanish. Even if they have extremely massive black holes $\left(10^{10} M_{\odot}\right)$, Elitzur \& Ho (2009) predict they would require radiatively inefficient accretion flows and bolometric luminosities $L_{\text {bol }}<5 \times 10^{41} \mathrm{erg} \mathrm{s}^{-1}$ for their BELRs to disappear; this scenario is excluded by their optical luminosities. Also, in the absence of a radiatively driven wind, the outflow is likely replaced by a jet, so we would expect very low accretion rate systems to also have strong radio emission (e.g., see Ho 2008).

It would be surprising if SDSS J0121-1020, SDSS J0901+0314, and 2QZJ 2154-3056 were relatively radio faint but otherwise normal BL Lac objects with their emission lines simply diluted by starlight from the host galaxy. Marchã et al. (1996) argue that the strongest emission features in weakly beamed BL Lac objects show equivalent widths up to $\sim 50-60 \AA$ when the equivalent width measures are referenced to the contaminating starlight continuum. In those cases, a boosted synchrotron continuum still acts to weaken the lines slightly (albeit less than if the AGN was more highly beamed). We do not believe the host galaxy on its own could render $\mathrm{H} \alpha$ or $\mathrm{H} \beta$ completely undetectable. In the absence of a boosted continuum, $\mathrm{H} \alpha$ and $\mathrm{H} \beta$ in these radio-quiet BL Lac candidates are likely intrinsically weak, but the mechanism is currently unclear (but see Londish et al. 2004 for potential explanations).

Finally, three other objects (SDSS J0256-0010, SDSS $\mathrm{J} 1048+6203$, and SDSS J2310-0005) remain as weak-featured AGNs, but we retain them with lower confidence because they have relatively noisy SDSS spectra. All three also lack spectroscopic redshifts, but SDSS J0256-0010 and SDSS J2310-0005 show variability in their Stripe 82 light curves. Both of these sources are also confirmed to be radio quiet in their deeper VLA observations. We do not have VLA data for SDSS J1048+6203, and its FIRST limit is not terribly sensitive $\left(\alpha_{\text {ro }}<0.281\right)$. It is possible this source is a normal radio-loud BL Lac object, but deeper radio and X-ray observations are necessary to settle this issue.

\subsection{Radio-quiet BL Lac Candidates from P10}

There are 71 additional $z<2.2$ objects identified as radioquiet BL Lac candidates in P10. All have FIRST/NVSS radio flux densities or limits in the radio-quiet regime $\left(\alpha_{\text {ro }}<0.2\right)$, so they are not just optically faint BL Lac objects with radio fluxes below the FIRST/NVSS limits. All but five of their objects have extragalactic redshifts, so there appears to be little contamination from stars. P10 argue that many of these objects are distinct from BL Lac objects because they tend to be at systematically higher redshifts and are more luminous than radio-loud BL Lac objects. However, deeper X-ray observations are necessary: the majority (95\%) do not have RASS X-ray detections, and their RASS flux limits are not sensitive enough to discriminate them from BL Lac objects in the X-ray. Based on our conclusions from our analysis of the C05/A07 sample, we expect that deeper X-ray observations will show the majority of P10 objects to be low-redshift WLQs.

\section{CONCLUSIONS}

Here, we present follow-up radio and X-ray observations for a subset of $z<2.2$ radio-quiet BL Lac candidates from a sample of 26 sources originally presented in C05 and A07. When available, we also consider flux variability and proper motion measures, and we conclude that 13 of the 26 objects are AGNs with weak emission lines. All but 3 of these 13 are definitively radio fainter than BL Lac objects at the $\geqslant 2.75 \sigma$ level (the other 3 objects are not detected in the radio, but their radio flux limits are not sensitive enough to declare them radio quiet), making it difficult to interpret them as beamed radio galaxies. We thus confirm that the SDSS has recovered a population of radio-quiet AGNs with intrinsically weak emission lines. That is, relativistic beaming does not appear to be the dominant cause of these objects' featureless spectra (but we also cannot exclude the possibility that weak jets exist in some of these sources). These 13 weak-featured AGNs are likely a heterogeneous mix of at least two populations of objects.

Of the 13 AGNs, 3 with deeper Chandra X-ray observations are likely low-redshift WLQs, analogs to the $\sim 10^{2}$ object high-redshift $(z>2.2)$ population discovered by the SDSS. Other low-redshift WLQ candidates have been discovered serendipitously in the SDSS (e.g., Hryniewicz et al. 2010), but the C05/A07 BL Lac surveys were the first systematic searches of the SDSS database capable of recovering low-redshift WLQs.

There are two radio-quiet BL Lac candidates that have X-ray detections in RASS and are too X-ray bright to be unified with WLQs, but their $\alpha_{\mathrm{ox}}^{\text {rass }}$ measures are similar to those of BL Lac objects. We retain these as radio-quiet BL Lac candidates, but with low confidence. One object's radio flux limit is not sensitive enough to declare it radio quiet (i.e., it could be a relatively radio faint but still normal radio-loud BL Lac object). The other object is definitively radio quiet, but its optical spectrum has a relatively low signal-to-noise ratio $(\mathrm{S} / \mathrm{N})$. The fact that we do not find a single high-confidence radio-quiet BL Lac object supports the notion that all BL Lac objects are radio-loud (Stocke et al. 1990).

There are eight other C05/A07 radio-quiet BL Lac candidates lacking RASS X-ray detections for which we do not have deeper Chandra coverage. It is likely some of these will also be shown to be low-redshift WLQs upon more detailed X-ray scrutiny. Many radio-quiet BL Lac candidates in P10 are probably also WLQs, and we thus conclude that the SDSS has discovered a new population of low-redshift WLQs.

We compare our radio-quiet SDSS BL Lac candidates to other radio-quiet BL Lac candidates/WLQs in the literature. We discuss how high-accretion rates can lead to soft ionizing continua and yield spectra with weak or absent UV lines but relatively strong $\mathrm{H} \alpha$ and $\mathrm{H} \beta$ (but those lines may still 
be weak compared to normal Type 1 quasars). Near-infrared spectroscopy is required to test this properly. Two of the eight objects lacking sensitive X-ray limits are similar to the radioquiet BL Lac candidate discovered by Londish et al. (2004, 2QZJ2154-3056). These objects are unlikely to have high-mass accretion rates (because they do not show $\mathrm{H} \beta$ ), and they are too luminous to have weak BELRs because of extraordinarily low accretion rates.

The radio-quiet BL Lac candidates should be monitored for polarization to determine if these objects show signs of weakly beamed relativistic jets. Objects with relatively larger (smaller) $\alpha_{\text {ro }}\left(\alpha_{\text {ox }}\right)$ values or limits similar to those for radio-loud BL Lac objects should be placed at high priority for polarimetric monitoring. Similarly, searches for flux variability and deeper radio observations for objects with poorer $\alpha_{\text {ro }}$ constraints would be useful. The detection of hot thermal dust emission in the infrared would strongly argue against a boosted continuum, as beaming would dilute dust emission (e.g., see Diamond-Stanic et al. 2009).

Londish et al. (2004) successfully model the spectrum of 2QZJ 2154-3056 as the combination of an elliptical galaxy, a starburst, and a central AGN, and an image decomposition shows a host galaxy and AGN. Their optical image cannot discriminate between an elliptical or disk host. Interestingly, PHL 1811 appears to live in a spiral galaxy (Jenkins et al. 2005). BL Lac objects are hosted (perhaps exclusively) by giant elliptical galaxies (e.g., see Urry et al. 2000). Thus, host galaxy imaging, which could be achieved at least for the lowest-redshift radio-quiet BL Lac candidates, might be a useful diagnostic.

The SDSS has discovered a population of at least 13 lowredshift $(z<2.2)$ radio-quiet AGNs with weak-featured optical spectra, many of which we believe are low-redshift analogs to WLQs. The P10 optically selected BL Lac sample adds up to another 71 objects. The case of SDSS J0945+1009 (Hryniewicz et al. 2010) with weak $\mathrm{Mg}$ II REW $\sim 15 \AA$ suggests there are likely even more objects yet to be recognized in the SDSS database (as SDSS BL Lac searches exclude objects with any emission feature larger than $5 \AA$ rest frame). In all likelihood, the SDSS radio-quiet BL Lac candidates are a heterogeneous mix of at least two populations of objects, so it is going to take even more observational effort to disentangle the physical nature of these strange objects.

We thank Adrienne Stilp for her assistance in reducing the VLA data, and we also thank the anonymous referee for helpful comments to improve this manuscript. This research has made use of software provided by the Chandra X-ray Center (CXC) in the application packages CIAO. R.M.P. and S.F.A. gratefully acknowledge support from NASA/ADP grant NNG05GC45G, and from the National Aeronautics and Space Administration through Chandra Award Number GO9-0126X issued by the Chandra X-ray Observatory Center, which is operated by the Smithsonian Astrophysical Observatory for and on behalf of the National Aeronautics Space Administration under contract NAS8-3060. W.N.B. acknowledges support from NASA/ADP grant NNX10AC99G.

Funding for the SDSS and SDSS-II has been provided by the Alfred P. Sloan Foundation, the Participating Institutions, the National Science Foundation, the U.S. Department of Energy, the National Aeronautics and Space Administration, the Japanese Monbukagakusho, the Max Planck Society, and the Higher Education Funding Council for England. The SDSS Web site is http://www.sdss.org/.
The SDSS is managed by the Astrophysical Research Consortium for the Participating Institutions. The Participating Institutions are the American Museum of Natural History, Astrophysical Institute Potsdam, University of Basel, University of Cambridge, Case Western Reserve University, University of Chicago, Drexel University, Fermilab, the Institute for Advanced Study, the Japan Participation Group, Johns Hopkins University, the Joint Institute for Nuclear Astrophysics, the Kavli Institute for Particle Astrophysics and Cosmology, the Korean Scientist Group, the Chinese Academy of Sciences (LAMOST), Los Alamos National Laboratory, the Max-PlanckInstitute for Astronomy (MPIA), the Max-Planck-Institute for Astrophysics (MPA), New Mexico State University, Ohio State University, University of Pittsburgh, University of Portsmouth, Princeton University, the United States Naval Observatory, and the University of Washington.

\section{REFERENCES}

Abazajian, K., et al. 2009, ApJS, 182, 543

Agüeros, M. A., et al. 2009, ApJS, 181, 444

Aller, M. F., Aller, H. D., Hughes, P. A., \& Latimer, G. E. 1999, ApJ, 512, 601 Anderson, S. F., et al. 2001, AJ, 122, 503

Anderson, S. F., et al. 2007, AJ, 133, 313 (A07)

Becker, R. H., White, R. L., \& Helfand, D. J. 1995, ApJ, 450, 559

Blandford, R. D., \& Rees, M. J. 1978, in Pittsburgh Conf. on BL Lac Objects, ed. A. M. Wolfe (Pittsburgh: Univ. Pittsburgh Press), 328

Blundell, K. M., Beasley, A. J., \& Bicknell, G. V. 2003, ApJ, 591, L103

Brinkmann, W., Laurent-Muehleisen, S. A., Voges, W., Siebert, J., Becker, R. H., Brotherton, M. S., White, R. L., \& Gregg, M. D. 2000, A\&A, 356, 445 Collinge, M. J., et al. 2005, AJ, 129, 2542 (C05)

Condon, J. J., Cotton, W. D., Greisen, E. W., Yin, Q. F., Perley, R. A., Taylor, G. B., \& Broderick, J. J. 1998, AJ, 115, 1693

Diamond-Stanic, A. M., et al. 2009, ApJ, 699, 782

Elitzur, M., \& Ho, L. C. 2009, ApJ, 701, L91

Fan, X., et al. 1999, ApJ, 526, L57

Fan, X., et al. 2006, AJ, 131, 1203

Garmire, G. P., Bautz, M. W., Ford, P. G., Nousek, J. A., \& Ricker, G. R. 2003, Proc. SPIE, 4851, 28

Gehrels, N. 1986, ApJ, 303, 336

Goto, T. 2005, MNRAS, 357, 937

Hawkins, M. R. S. 2004, A\&A, 424, 519

Ho, L. C. 2008, ARA\&A, 46, 475

Hryniewicz, K., Czerny, B., Nikolajuk, M., \& Kuraszkiewicz, J. 2010, MNRAS, 404, 2028

Ivezić, Ž., et al. 2007, AJ, 134, 973

Jannuzi, B. T., Green, R. F., \& French, H. 1993, ApJ, 404, 100

Jenkins, E. B., Bowen, D. V., Tripp, T. M., \& Sembach, K. R. 2005, ApJ, 623, 767

Just, D. W., Brandt, W. N., Shemmer, O., Steffen, A. T., Schneider, D. P., Chartas, G., \& Garmire, G. P. 2007, ApJ, 665, 1004

Kellermann, K. I., Sramek, R., Schmidt, M., Shaffer, D. B., \& Green, R. 1989, AJ, 98, 1195

Kelly, B. C., Bechtold, J., \& Siemiginowska, A. 2009, ApJ, 698, 895

Kollgaard, R. I. 1994, Vistas Astron., 38, 29

Landt, H., Padovani, P., \& Giommi, P. 2002, MNRAS, 336, 945

Leighly, K. M., Halpern, J. P., Jenkins, E. B., \& Casebeer, D. 2007a, ApJS, 173,1

Leighly, K. M., Halpern, J. P., Jenkins, E. B., Grupe, D., Choi, J., \& Prescott, K. B. 2007b, ApJ, 663, 103

Londish, D., Heidt, J., Boyle, B. J., Croom, S. M., \& Kedziora-Chudczer, L. 2004, MNRAS, 352, 903

MacLeod, C. L., et al. 2010, ApJ, submitted (arXiv:1004.0276)

Mannucci, F., Basile, F., Poggianti, B. M., Cimatti, A., Daddi, E., Pozzetti, L., \& Vanzi, L. 2001, MNRAS, 326, 745

Marchã, M. J. M., Browne, I. W. A., Impey, C. D., \& Smith, P. S. 1996, MNRAS, 281,425

Marscher, A. P., \& Gear, W. K. 1985, ApJ, 298, 114

Massaro, E., Giommi, P., Leto, C., Marchegiani, P., Maselli, A., Perri, M., Piranomonte, S., \& Sclavi, S. 2009, A\&A, 495, 691

McDowell, J. C., Canizares, C., Elvis, M., Lawrence, A., Markoff, S., Mathur, S., \& Wilkes, B. J. 1995, ApJ, 450, 585

Mukai, K. 1993, Legacy, 3, 21 
Munn, J. A., et al. 2004, AJ, 127, 3034

Nicastro, F. 2000, ApJ, 530, L65

Nicastro, F., Martocchia, A., \& Matt, G. 2003, ApJ, 589, L13

Padovani, P., \& Giommi, P. 1995a, ApJ, 444, 567

Padovani, P., \& Giommi, P. 1995b, MNRAS, 277, 1477

Perlman, E. S., Padovani, P., Landt, H., Stocke, J. T., Costamante, L., Rector, T., Giommi, P., \& Schachter, J. F. 2001, in ASP Conf. Ser. 227, Blazar Demographics and Physics, ed. P. Padovani \& C. M. Urry (San Francisco, CA: ASP), 200

Plotkin, R. M., Anderson, S. F., Hall, P. B., Margon, B., Voges, W., Schneider, D. P., Stinson, G., \& York, D. G. 2008, AJ, 135, 2453

Plotkin, R. M., et al. 2010, AJ, 139, 390 (P10)

Schlegel, D. J., Finkbeiner, D. P., \& Davis, M. 1998, ApJ, 500, 525

Schneider, D. P., et al. 2007, AJ, 134, 102

Schneider, D. P., et al. 2010, AJ, 139, 2360

Sesar, B., et al. 2007, AJ, 134, 2236

Shemmer, O., Brandt, W. N., Anderson, S. F., Diamond-Stanic, A. M., Fan, X., Richards, G. T., Schneider, D. P., \& Strauss, M. A. 2009, ApJ, 696, 580

Shemmer, O., Netzer, H., Maiolino, R., Oliva, E., Croom, S., Corbett, E., \& di Fabrizio, L. 2004, ApJ, 614, 547

Shemmer, O., et al. 2006, ApJ, 644, 86

Smith, P. S., Williams, G. G., Schmidt, G. D., Diamond-Stanic, A. M., \& Means, D. L. 2007, ApJ, 663, 118
Stark, A. A., et al. 1992, ApJS, 79, 77

Steffen, A. T., Strateva, I., Brandt, W. N., Alexander, D. M., Koekemoer, A. M., Lehmer, B. D., Schneider, D. P., \& Vignali, C. 2006, AJ, 131, 2826

Stickel, M., Padovani, P., Urry, C. M., Fried, J. W., \& Kuehr, H. 1991, ApJ, 374, 431

Stocke, J. T., Liebert, J., Schmidt, G., Gioia, I. M., Maccacaro, T., Schild, R. E., Maccagni, D., \& Arp, H. C. 1985, ApJ, 298, 619

Stocke, J. T., Morris, S. L., Weymann, R. J., \& Foltz, C. B. 1992, ApJ, 396, 487

Stocke, J. T., Morris, S. L., Gioia, I., Maccacaro, T., Schild, R. E., \& Wolter, A. 1990, ApJ, 348, 141

Stocke, J. T., Morris, S. L., Gioia, I. M., Maccacaro, T., Schild, R., Wolter, A., Fleming, T. A., \& Henry, J. P. 1991, ApJS, 76, 813

Tananbaum, H., et al. 1979, ApJ, 234, L9

Trump, J. R., et al. 2009, ApJ, 706, 797

Trump, J. R., et al. 2006, ApJS, 165, 1

Ulrich, M.-H., Maraschi, L., \& Urry, C. M. 1997, ARA\&A, 35, 445

Urry, C. M., \& Padovani, P. 1995, PASP, 107, 803

Urry, C. M., Scarpa, R., O’Dowd, M., Falomo, R., Pesce, J. E., \& Treves, A. 2000, ApJ, 532, 816

Vanden Berk, D. E., et al. 2001, AJ, 122, 549

Voges, W., et al. 1999, A\&A, 349, 389

Voges, W., et al. 2000, IAU Circ., 7432

York, D. G., et al. 2000, AJ, 120, 1579 